\title{
Evaluation of candidate geomagnetic field models for IGRF-11
}

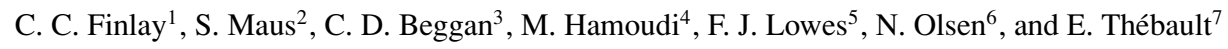 \\ ${ }^{1}$ Earth and Planetary Magnetism Group, Institut für Geophysik, Sonneggstrasse 5, ETH Zürich, CH-8092, Switzerland \\ ${ }^{2}$ NOAA/NGDC and CIRES, University of Colorado, U.S.A. \\ ${ }^{3}$ British Geological Survey, Murchison House, West Mains Road, Edinburgh, EH9 3LA, U.K. \\ ${ }^{4}$ Helmholtz Centre Potsdam, GFZ German Research centre for Geosciences, Telegrafenberg, 14473, Germany \\ ${ }^{5}$ School of Chemistry, University of Newcastle, Newcastle Upon Tyne, NE1 7RU, U.K. \\ ${ }^{6}$ DTU Space, Juliane Maries Vej 30, 2100 Copenhagen, Denmark \\ ${ }^{7}$ Équipe de Géomagnétisme, Institut de Physique du Globe de Paris, UMR 7154, CNRSINSU, Univ. Paris Diderot, Paris, France
}

(Received April 20, 2010; Revised September 24, 2010; Accepted November 18, 2010; Online published December 31, 2010)

\begin{abstract}
The eleventh generation of the International Geomagnetic Reference Field (IGRF) was agreed in December 2009 by a task force appointed by the International Association of Geomagnetism and Aeronomy (IAGA) Division V Working Group V-MOD. New spherical harmonic main field models for epochs 2005.0 (DGRF-2005) and 2010.0 (IGRF-2010), and predictive linear secular variation for the interval 2010.0-2015.0 (SV-2010-2015) were derived from weighted averages of candidate models submitted by teams led by DTU Space, Denmark (team A); NOAA/NGDC, U.S.A. (team B); BGS, U.K. (team C); IZMIRAN, Russia (team D); EOST, France (team E); IPGP, France (team F); GFZ, Germany (team G) and NASA-GSFC, U.S.A. (team H). Here, we report the evaluations of candidate models carried out by the IGRF-11 task force during October/November 2009 and describe the weightings used to derive the new IGRF-11 model. The evaluations include calculations of root mean square vector field differences between the candidates, comparisons of the power spectra, and degree correlations between the candidates and a mean model. Coefficient by coefficient analysis including determination of weighting factors used in a robust estimation of mean coefficients is also reported. Maps of differences in the vertical field intensity at Earth's surface between the candidates and weighted mean models are presented. Candidates with anomalous aspects are identified and efforts made to pinpoint both troublesome coefficients and geographical regions where large variations between candidates originate. A retrospective analysis of IGRF-10 main field candidates for epoch 2005.0 and predictive secular variation candidates for 2005.0-2010.0 using the new IGRF-11 models as a reference is also reported. The high quality and consistency of main field models derived using vector satellite data is demonstrated; based on internal consistency DGRF-2005 has a formal root mean square vector field error over Earth's surface of $1.0 \mathrm{nT}$. Difficulties nevertheless remain in accurately forecasting field evolution only five years into the future.
\end{abstract}

Key words: Geomagnetism, field modelling, reference field, secular variation.

\section{Introduction}

The IGRF is an internationally agreed spherical harmonic reference model describing the largest scales of the internal part of the Earth's magnetic field. It is widely used by scientists studying local and regional crustal magnetic anomalies, by those studying space weather and solar-terrestrial magnetic interactions, and it is also sometimes used by individuals and commercial organizations for navigational purposes. Under normal circumstances the IGRF is updated every 5 years; for a history of IGRF and further background information consult Barton (1997), or Macmillan and Finlay (2010). An IGRF update involves collaboration between institutes collecting and disseminating geomagnetic measurements derived from satellites and ground-based observatories, and between teams of geomagnetic field modellers, making it a truly international enterprise.

Copyright (C) The Society of Geomagnetism and Earth, Planetary and Space Sciences (SGEPSS); The Seismological Society of Japan; The Volcanological Society of Japan; The Geodetic Society of Japan; The Japanese Society for Planetary Sciences; TERRAPUB.

doi:10.5047/eps.2010.11.005
Ultimate responsibility for producing an updated IGRF model lies with IAGA. At a business meeting of IAGA Division V Working Group V-MOD (hereafter referred to as IAGA Div V, WG V-MOD) in Perugia in July 2007, a task force with responsibility for the production of IGRF-11 was elected. This consisted of C. Finlay (Chair, ETHZ), S. Maus/S. McLean (NGDC), F. Lowes (Univ. Newcastle), N. Olsen (DTU Space), A. Chambodut (EOST), V. Lesur (GFZ), E. Thébault (IPGP), T. Sabaka (NASA), T. Bondar (IZMIRAN) and S. Macmillan (BGS). The task force included only one voting member from each institution contributing candidate models; this permitted the operation of a democratic voting system to make the necessary collective decisions. For example, in April 2009 the task force voted to retain a spherical harmonic truncation degree of 8 for the predictive secular variation (SV) in IGRF-11. In May 2009 a call for IGRF-11 candidate models was agreed on by the task force and issued. This requested main field (MF) candidate models for the Definitive Geomagnetic Reference Field for epoch 2005.0 (DGRF-2005), for a provisional IGRF model for epoch 2010.0 (IGRF-2010) both to spherical har- 
monic degree 13 , and for a prediction of the average SV over the upcoming five years (SV-2010-2015) to degree 8. An update of progress towards IGRF-11 was given by the task force chair at a business meeting of IAGA Div V, WG V-MOD in Sopron in August 2009.

At the start of October 2009, seven MF candidate models were submitted for DGRF-2005 and IGRF-2010, while eight candidates were submitted for SV-2010-2015. Following a vote by the task force it was decided to allow teams to resubmit revised candidate models before the end of October 2009, due to problems with some initial candidates. BGS submitted revised candidate models for all three products, EOST submitted a revised DGRF candidate and IZMIRAN submitted a late DGRF candidate during this period. During November 2009 members of the task force and other interested parties carried out evaluations of the candidate models and submitted proposals concerning how the candidates should be weighted in the derivation of IGRF-11. Ten independent evaluations were received and posted online for consideration by the task force members. Following internal discussions within the task force, the task force chair (in consultation with the IAGA Div V, WG V-MOD chair) prepared a ballot paper containing various weighting options. This was voted on by the task force and the results announced on 7th December 2009. The final coefficients were prepared and checked, before being made available to the public through the IAGA Div V, WG V-MOD webpage http://www. ngdc.noaa.gov/IAGA/vmod/igrf.html on 24th December 2009. A summary of the construction of IGRF-11 will be presented at the next business meeting of IAGA Div V, WG V-MOD in Melbourne in July 2011. Further details about the process including progress reports, candidate models and descriptions provided by the authors, original evaluations and test models designed to aid decisions regarding IGRF-12, can be found at http://www. ngdc.noaa.gov/IAGA/vmod/candidatemodels.html.

The primary sources of data employed by the modelling teams to produce candidate models were from the German satellite CHAMP, the Danish satellite Ørsted and the Argentine-U.S.-Danish satellite SAC-C, along with data from the international network of geomagnetic observatories. The teams adopted a variety of data selection and processing procedures. Furthermore, the required single epoch spherical harmonic model coefficients were derived from parent models that used a range of time durations (1 month to 12 years), temporal parameterizations (including Taylor series of degree 0 to 2 , splines of order 1 to 6 ), and external field parameterizations of varying complexity. The parent models also used a number of alternative parameter estimation schemes (including least-squares, least absolute deviations, robust estimation based on Huber's distribution and natural orthogonal analysis). Further details concerning the techniques used to derive the individual candidate models can be found in the papers appearing in this special issue (Chambodut et al., 2010; Hamilton et al., 2010; Kuang et al., 2010; Lesur et al., 2010; Maus et al., 2010; Olsen et al., 2010; Thébault et al., 2010). The different strategies adopted naturally lead to differences in the submitted candidate models. As described above, the task force there- fore undertook testing and inter-comparison of the candidates to produce the information required for decisions on the weights to be used in the construction of IGRF-11.

The purpose of the present article is to summarize the evaluations of candidate models carried out in October/November 2009 by the IGRF-11 task force, and to report the final weighting of the candidate models used to derive IGRF-11. We follow closely the strategy adopted in previous evaluations (see, for example, Maus et al., 2005 ) focusing on statistical comparisons between the candidate models and various mean models, and utilizing wellestablished diagnostic tools in both the spectral and physical domains. One limitation of this approach is that a good statistical agreement between models does not necessarily mean these models are the most realistic; it can also be a consequence of the use of very similar data selection or modelling techniques. Model evaluations would ideally be based not only upon statistical analysis of candidates, but also on comparisons with independent data that accurately measured the relevant field (the internal magnetic field at Earth's surface) at the epochs of interest. Unfortunately such ideal evaluation data did not exist for the future epochs of 2010.0 and 2010.0-2015.0 at the time of the evaluations, and it is even troublesome to obtain high quality independent data for the retrospective epoch 2005.0. Attempts to assess the candidate models using either observatory or satellite data are thus complicated by the necessity of propagating the models to suitable comparison epochs as well as with difficulties in separating internal and external field contributions in the observed data. Nonetheless, some workers have made interesting attempts at such comparisons, see for example the study by Chulliat and Thébault (2010) in this issue.

As a mathematical preliminary, we begin in Section 2 by providing the formulae defining the analysis tools employed. In Section $3 \mathrm{MF}$ candidates are studied, while Section 4 presents evaluations of SV candidates. In Section 3.1 we analyze the candidate models for DGRF2005 , then in Section 3.2 a retrospective evaluation of the IGRF-10 candidates for epoch 2005 in comparison with the new DGRF-2005 model is carried out. In Section 3.3 evaluations of the candidates for IGRF-2010 are presented, followed in Section 4.1 by a retrospective analysis of the predictive SV candidates for the epoch 2005-2010 from IGRF10. Finally in Section 4.2 the IGRF-11 predictive SV candidates for epoch 2010-2015 are analyzed. In each case global comparisons of root mean square (RMS) vector field differences are made first, then comparisons in the spectral domain, per degree and then coefficient by coefficient; finally maps of differences between candidate models and a weighted mean model are presented. Discussion of the evaluation results and a summary of the decision of the task force is provided for each IGRF-11 product. We conclude with an overall summary and some remarks on the implications of these evaluations for the future of the IGRF.

\section{Mathematical Definitions and Formulae Used in Evaluations}

Formulae defining the diagnostic tools employed during the evaluations are first presented to avoid ambigu- 
ity. The IGRF-11 candidate models take the form of Schmidt semi-normalized (sometimes also referred to as quasi-normalized) spherical harmonic coefficients (see, for example, Winch et al., 2004) with units of nT for MF models and nT/yr for SV models. In what follows $g_{n}^{m}$ and $h_{n}^{m}$ are used to denote the spherical harmonic coefficients associated with the $\cos m \phi$ and $\sin m \phi$ components respectively, where $\phi$ denotes geocentric longitude. As is conventional $n$ denotes spherical harmonic degree while $m$ denotes spherical harmonic order. Often we will be concerned with differences between a candidate model $i$ whose coefficients we denote by ${ }_{i} g_{n}^{m}$ and ${ }_{i} h_{n}^{m}$ and some other reference model (labelled $j$ ) whose coefficients will be denoted by ${ }_{j} g_{n}^{m}$ and ${ }_{j} h_{n}^{m}$. It is also convenient at this point to define the difference between the coefficients of two such models as

$$
{ }_{i, j} g_{n}^{m}={ }_{i} g_{n}^{m}-{ }_{j} g_{n}^{m} \quad \text { and } \quad{ }_{i, j} h_{n}^{m}={ }_{i} h_{n}^{m}-{ }_{j} h_{n}^{m} .
$$

Much use will be made below of the mean square vector field difference between models per spherical harmonic degree ${ }_{i, j} R_{n}$ (see, for example, Lowes $(1966,1974)$ )

$$
{ }_{i, j} R_{n}=(n+1)\left(\frac{a}{r}\right)^{(2 n+4)} \sum_{m=0}^{n}\left[\left(i, j g_{n}^{m}\right)^{2}+\left({ }_{i, j} h_{n}^{m}\right)^{2}\right]
$$

where $a$ is the magnetic reference spherical radius of $6371.2 \mathrm{~km}$ which is close to the mean Earth radius, and $r$ is the radius of the sphere of interest, which is taken as $r=a$ for comparisons at the Earth's surface and $r=3480 \mathrm{~km}$ for comparisons at the core-mantle boundary. Taking the special case when the reference model is zero, denoted by 0 , (2) reduces to the standard Lowes-Mauersberger geomagnetic power spectrum $_{i, 0} R_{n}$ for a given model $i$

$$
{ }_{i, 0} R_{n}=(n+1)\left(\frac{a}{r}\right)^{(2 n+4)} \sum_{m=0}^{N}\left[\left(g_{n}^{m}\right)^{2}+\left(h_{n}^{m}\right)^{2}\right] .
$$

Summing over degrees $n$ from 1 to the truncation degree $N$ and taking the square root yields the RMS vector field difference between the models $i$ and $j$ averaged over the spherical surface

$$
i, j R=\sqrt{\sum_{n=1}^{N} i, j} R_{n}
$$

It is sometimes informative to calculate $i, j R$ when the reference model $j$ is a weighted mean of the $K$ candidates models with each model allocated a weight ${ }_{i} w$. The coefficients of the weighted mean model $M_{w}$ are then

$$
\widetilde{g_{n}^{m}}=\frac{\sum_{i=1}^{K}{ }_{i} w_{i} g_{n}^{m}}{\sum_{i=1}^{K}{ }_{i} w} \text { and } \widetilde{h_{n}^{m}}=\frac{\sum_{i=1}^{K}{ }_{i} w_{i} h_{n}^{m}}{\sum_{i=1}^{K}{ }_{i} w}
$$

The precise details of the weightings used will be discussed in detail below. In the special case when all ${ }_{i} w=1$ we obtain the simple arithmetic mean model (which we refer to below as model $M$ ) with coefficients

$$
\overline{g_{n}^{m}}=\frac{1}{K} \sum_{i=1}^{K}{ }_{i} g_{n}^{m} \quad \text { and } \quad \overline{h_{n}^{m}}=\frac{1}{K} \sum_{i=1}^{K}{ }_{i} h_{n}^{m} .
$$

In addition to calculating ${ }_{i, j} R$ for individual models, it is also possible to compute the mean value of ${ }_{i, j} R$ for the $i$ th model compared to the $(K-1)$ other candidates labelled by $j$, such that

$$
{ }_{i} \bar{R}=\frac{1}{(K-1)} \sum_{\text {candidates }}{ }_{j \neq i} R \text {. }
$$

Analysis of spherical harmonic spectra is a powerful way to diagnose differences in amplitude between models but tells us little about how well they are correlated. The correlation per degree between two models, again labelled by the indices $i$ and $j$, can be studied as a function of spherical harmonic degree using the quantity $i, j \rho_{n}$ (see, for example p. 81 of Langel and Hinze (1998))

$$
i_{, j} \rho_{n}=\frac{\sum_{m=0}^{n}\left({ }_{i} g_{n j}^{m} g_{n}^{m}+{ }_{i} h_{n j}^{m} h_{n}^{m}\right)}{\sqrt{\left(\sum_{m=0}^{n}\left[\left({ }_{i} g_{n}^{m}\right)^{2}+\left({ }_{i} h_{n}^{m}\right)^{2}\right]\right)\left(\sum_{m=0}^{n}\left[\left({ }_{j} g_{n}^{m}\right)^{2}+\left({ }_{j} h_{n}^{m}\right)^{2}\right]\right)}} .
$$

The degree correlation between a model $i$ and the arithmetic mean model $M$ that is frequently considered below may then be defined as

$$
i, M \rho_{n}=\frac{\sum_{m=0}^{n}\left({ }_{i} g_{n}^{m} \overline{g_{n}^{m}}+{ }_{i} h_{n}^{m} \overline{h_{n}^{m}}\right)}{\sqrt{\left(\sum_{m=0}^{n}\left[\left({ }_{i} g_{n}^{m}\right)^{2}+\left({ }_{i} h_{n}^{m}\right)^{2}\right]\right)\left(\sum_{m=0}^{n}\left[\left(\overline{g_{n}^{m}}\right)^{2}+\left(\overline{h_{n}^{m}}\right)^{2}\right]\right)}} .
$$

Assuming that the candidate models are independent, that they involve only random errors, and that these errors have a standard deviation at degree $n$ common to all the $K$ contributing models, then this common sample standard deviation can be estimated from the scatter about the mean. Expressed in terms of a per degree sample standard deviation $s_{n}$, the RMS scatter of the vector magnetic field over the reference sphere (as derived from Eq. (3)) is given by

$$
s_{n}=\sqrt{\frac{n-1}{K-1} \sum_{i=1}^{K} \sum_{m=0}^{n}\left({ }_{i} g_{n}^{m}-\overline{g_{n}^{m}}\right)^{2}+\left({ }_{i} h_{n}^{m}-\overline{h_{n}^{m}}\right)^{2}} .
$$

The corresponding standard error in the arithmetic mean determined from these $K$ models is then

$$
e_{n}=\frac{s_{n}}{\sqrt{K}}
$$

A final statistical tool of interest is the method of 'robust' estimation (see, for example, Hogg, 1979; Huber, 1996). This approach is known to be of value when error distributions are non-Gaussian, in particular if outliers are present. During the IGRF-11 evaluation process, in an investigation of the applicability of this method, the 'robust' weighted mean of each spherical harmonic coefficient was determined by treating the set of values for each coefficient (i.e. the $K$ values for each ${ }_{i} g_{n}^{m}$ or ${ }_{i} h_{n}^{m}$ ) independently. The 
Table 1. Summary of DGRF-2005 candidate models submitted to IGRF-11.

\begin{tabular}{|c|c|c|c|c|}
\hline \multicolumn{5}{|c|}{ DGRF candidate models for main field epoch 2005} \\
\hline Team & Model & Organization & Data & Comments (parent model etc.) \\
\hline A & DGRF-2005-A & $\begin{array}{c}\text { DTU Space / } \\
\text { IPGP / GSFC-NASA }\end{array}$ & $\begin{array}{l}\text { Ørsted, CHAMP, SAC-C } \\
\text { revised observatory monthly means }\end{array}$ & $\begin{array}{l}\text { Based on CHAOS- } 3 \alpha \text { in } 2005.0 \\
\text { (6th order splines for parent) }\end{array}$ \\
\hline B & DGRF-2005-B & NGDC-NOAA / GFZ & CHAMP 2003.5-2006.5 & $\begin{array}{l}\text { Based on POMME } 6 \\
\text { 2nd order Taylor series }\end{array}$ \\
\hline $\mathrm{C}$ & DGRF-2005-C2 & BGS & $\begin{array}{l}\text { Ørsted, CHAMP and observatory hourly means } \\
\text { for 01:00-02:00 LT, 1999.0-2009.5 }\end{array}$ & $\begin{array}{c}\text { Revised submission: parent model } \\
\text { linear splines (400 day knots spacing) }\end{array}$ \\
\hline $\mathrm{D}$ & DGRF-2005-D & IZMIRAN & $\begin{array}{c}\text { CHAMP 2004.0-2006.0 } \\
\text { no data selection } \\
\end{array}$ & $\begin{array}{c}\text { Natural Orthogonal Components (NOC) } \\
\text { method with } 5 \text { terms }\end{array}$ \\
\hline $\mathrm{E}$ & DGRF-2005-E2 & $\begin{array}{l}\text { EOST / LPGN / } \\
\text { / LATMOS / IPGP }\end{array}$ & $\begin{array}{l}\text { CHAMP \& Ørsted } \\
2004.5-2005.5\end{array}$ & $\begin{array}{l}\text { Revised submission: based on } \\
12 \text { month model with linear SV }\end{array}$ \\
\hline $\mathrm{F}$ & DGRF-2005-F & $\begin{array}{c}\text { IPGP / EOST } \\
\text { / LPGN / LATMOS }\end{array}$ & CHAMP 2004.4-2005.7 & 2nd order Taylor series (to $n=5$ ) \\
\hline G & DGRF-2005-G & GFZ & $\begin{array}{c}\text { CHAMP 2001-2009.6 } \\
\text { observatory hourly means }\end{array}$ & $\begin{array}{c}\text { Based on GRIMM2 } \\
\text { (6th order splines for parent) averaged over } 1 \mathrm{yr} \text {. }\end{array}$ \\
\hline
\end{tabular}

weights entering this calculation were determined by an error distribution known as the Huber distribution

$$
H(\epsilon)=\frac{1}{N_{c}} \begin{cases}\exp \left(-\epsilon^{2} / 2\right), & |\epsilon|<c \\ \exp (-c|\epsilon|+c / 2), & |\epsilon| \geq c\end{cases}
$$

where $\epsilon$ is the normalized departure from the mean, $c=$ 1.5 is a parameter chosen for a compromise between a Laplacian distribution (obtained when $c=0$ ) and a Gaussian distribution (obtained when $c \rightarrow \infty$ ), and $N_{c}=$ 2.6046 is a constant that ensures the correct normalization for the choice $c=1.5$. This distribution treats large departures from the mean as coming from a Laplacian distribution, thus avoiding undue influence on the parameter estimate. Maximum likelihood estimates of a robust mean with the errors assumed distributed as in (12) can conveniently be determined by an iteratively-reweighted least squares (IRLS) procedure (Constable, 1988; Olsen, 2002). In this method for the $q$ th iteration the weight for the $i$ th model for a given spherical harmonic coefficient labelled by $\alpha$ i.e. $\left({ }_{i} w_{\alpha}\right)_{q}$ is determined from the associated residuals from the current weighted mean ${ }_{i}\left(\epsilon_{\alpha}\right)_{q}$ such that

$$
\left({ }_{i} w_{\alpha}\right)_{q}=\min \left(c /\left|\left({ }_{i} \epsilon_{\alpha}\right)_{q}\right|, 1.0\right) .
$$

Below we plot the converged weights ${ }_{i} w_{\alpha}$ for each spherical harmonic coefficient of each candidate model, i.e. for all ${ }_{i} g_{m}^{n},{ }_{i} h_{m}^{n}$, in order to compare candidates. Coefficients allocated low weights are effectively identified as outliers under this scheme. However, note once again that this procedure treats each spherical harmonic coefficient $\alpha$ as independent, and the 'robust mean' coefficients neglect any prior information that may be gleaned from other coefficients. This may be particularly problematic if candidate models contain strongly correlated Gauss coefficients. Thus, we use the Huber weights only as a diagnosis tool and do not use them to determine the final weights given to the candidate models.

Having defined the tools used in the evaluations, we now proceed to present the results of the analysis, together with related discussion of the weightings allocated to candidates in the final IGRF-11 models.

\section{Evaluation of Main Field Candidate Models 3.1 Analysis of IGRF-11 DGRF-2005 candidate mod- els}

Table 1 lists the seven candidates models for DGRF 2005 giving details of the teams, the major data sources used and very brief comments concerning the various modelling approaches adopted. Two candidates (C2 and E2) were resubmissions as the original candidates were withdrawn by their authors.

3.1.1 RMS vector field differences for DGRF-2005 candidate models Rows of Table 2 present the RMS vector field differences ${ }_{i, j} R$ in units of $\mathrm{nT}$ between a particular DGRF candidate model $i$ and another candidate $j$. The final three columns document ${ }_{i, j} R$ between a candidate model $i$ and one of three possible mean models $j$. The mean models considered are the arithmetic mean model $M$, the model $M_{\text {noD }}$ which is an arithmetic mean excluding candidate $\mathrm{D}$, and the model $M_{\mathrm{ABG}}$ that is an arithmetic mean model derived only from candidates A, B and G. Note the symmetry about the diagonal entries in this table which is included as a check on the calculations. It is readily observed that model D is consistently furthest away from the other models in terms of $i, j R$; furthermore the RMS vector field differences between the other candidates and the mean are reduced when $\mathrm{D}$ is removed from the calculation of the mean. On the other hand candidates A, B, G are found to be extremely similar displaying the smallest RMS vector field differences between each other. Besides candidate D, candidates $\mathrm{C} 2$ and E2 show the next largest ${ }_{i, j} R$ followed by F.

The final three rows of Table 2 involve the arithmetic means of the RMS vector field differences of ${ }_{i, j} R$ of model $i$ from the other models $j$. The third from last row is ${ }_{i} \bar{R}$, the penultimate row is the same calculation excluding candidate $\mathrm{D}$ while the final row involves only ${ }_{i, j} R$ from candidates $\mathrm{A}$, $\mathrm{B}$ and $\mathrm{G}$. Candidates $\mathrm{A}, \mathrm{B}$ and $\mathrm{G}$ have the smallest ${ }_{i} \bar{R}$ and 
Table 2. RMS vector field differences ${ }_{i, j} R$ in units nT between DGRF-2005 candidate models and also between candidates and the arithmetic mean reference models $M, M_{\mathrm{noD}}$ and $M_{\mathrm{ABG}}$ shown in the rightmost columns. The bottom three rows are arithmetic means ${ }_{i} \bar{R}$ of the ${ }_{i, j} R$ where the means include respectively all candidates, exclude candidate D, and use only models A, B and G.

\begin{tabular}{|c|r|r|r|r|r|r|r||r|r|r|}
\hline \hline$i, j R / \mathrm{nT}$ & $\mathrm{A}$ & \multicolumn{1}{|c|}{$\mathrm{B}$} & $\mathrm{C} 2$ & \multicolumn{1}{|c|}{$\mathrm{D}$} & $\mathrm{E} 2$ & $\mathrm{~F}$ & $\mathrm{G}$ & $M$ & $M_{\mathrm{noD}}$ & $M_{\mathrm{ABG}}$ \\
\hline \hline $\mathrm{A}$ & 0.0 & 2.3 & 4.3 & 14.9 & 5.4 & 4.6 & 2.9 & 3.1 & 2.0 & 1.6 \\
\hline $\mathrm{B}$ & 2.3 & 0.0 & 4.8 & 14.5 & 5.2 & 3.8 & 2.2 & 2.6 & 1.7 & 1.2 \\
\hline $\mathrm{C} 2$ & 4.3 & 4.8 & 0.0 & 15.2 & 6.8 & 6.5 & 5.2 & 4.6 & 4.0 & 4.6 \\
\hline $\mathrm{D}$ & 14.9 & 14.5 & 15.2 & 0.0 & 14.6 & 15.0 & 14.4 & 12.4 & 14.4 & 14.5 \\
\hline $\mathrm{E} 2$ & 5.4 & 5.2 & 6.8 & 14.6 & 0.0 & 5.6 & 5.6 & 4.5 & 4.2 & 5.2 \\
\hline $\mathrm{F}$ & 4.6 & 3.8 & 6.5 & 15.0 & 5.6 & 0.0 & 4.4 & 4.1 & 3.4 & 4.0 \\
\hline $\mathrm{G}$ & 2.9 & 2.2 & 5.2 & 14.4 & 5.6 & 4.4 & 0.0 & 3.0 & 2.4 & 1.6 \\
\hline \hline Mean Diff & 5.7 & 5.5 & 7.1 & 14.7 & 7.2 & 6.6 & 5.8 & 4.9 & 4.6 & 4.7 \\
\hline Mean Diff noD & 3.9 & 3.7 & 5.5 & 17.7 & 5.7 & 5.0 & 4.1 & 3.7 & 3.0 & 3.0 \\
\hline Mean Diff ABG & 2.6 & 2.2 & 4.8 & 14.6 & 5.4 & 4.2 & 2.6 & 2.9 & 2.0 & 1.4 \\
\hline \hline
\end{tabular}
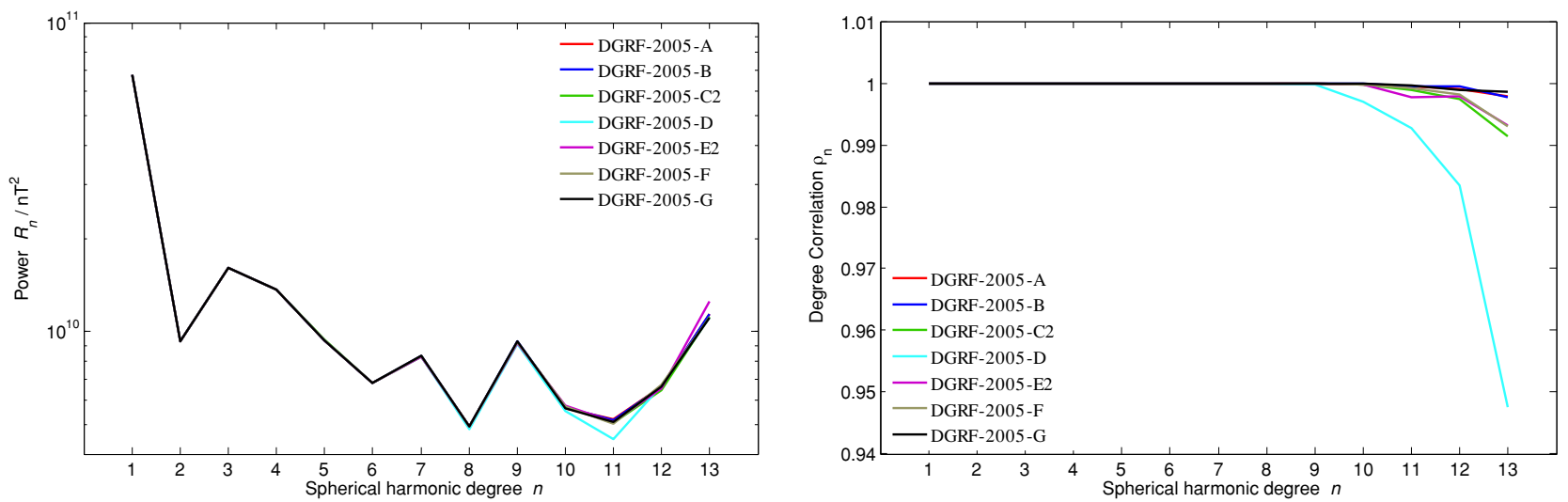

Fig. 1. Lowes-Mauersberger spectra ${ }_{i, 0} R_{n}$ from (3) of DGRF-2005 candidate models at radius $3480 \mathrm{~km}$ (core-mantle boundary) (left) and degree correlation $_{i, M} \rho_{n}$ from (9) between DGRF-2005 candidate models and their arithmetic mean model $M$ (right).
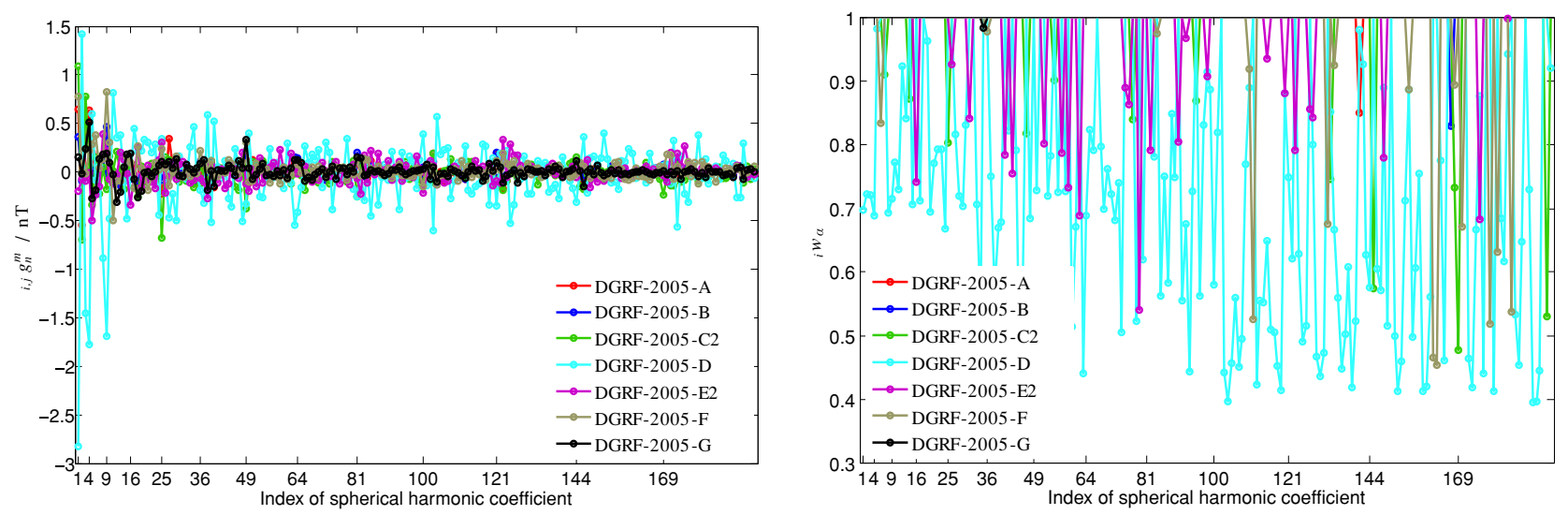

Fig. 2. Left plot shows differences ${ }_{i, j} g_{n}^{m}$ as defined in (1) between DGRF-2005 candidate models and their mean model $M$ as a function of the index of the spherical harmonic coefficient (running from $g_{1}^{0}, g_{1}^{1}, h_{1}^{1}, g_{2}^{0}, h_{1}^{1}$ etc indexed 1, 2, 3, 4, 5, etc). Right plot shows the Huber robust weighting factor ${ }_{i} w_{\alpha}$, where 1.0 indicates full weight 0.0 indicates zero weight, also as a function of the spherical harmonic coefficient.

the mean of the ${ }_{i, j} R$ becomes smaller when only candidates $\mathrm{A}, \mathrm{B}$ and $\mathrm{G}$ are retained.

3.1.2 Spectral analysis of DGRF-2005 candidate models Figure 1 (left) presents the Lowes-Mauersberger spectra ${ }_{i, 0} R_{n}$ (defined in (3)) of the DGRF-2005 candidate models as a function of spherical harmonic degree plotted at the Earth's core-mantle boundary $(r=3480 \mathrm{~km})$. The spectra of the candidate models are mostly very similar, al- most completely overlapping for degrees less than 9. The most noticeable differences occur for candidate $\mathrm{D}$ at degree 11 (where it contains lower power than the other candidates) and for candidate E2 at degree 13 (where it contains higher power than the other candidates). Figure 1 (right) presents the degree correlation $i, M \rho_{n}$ as defined in (9) between the DGRF-2005 candidate models and the arithmetic mean model $M$. Candidate D displays a low degree cor- 

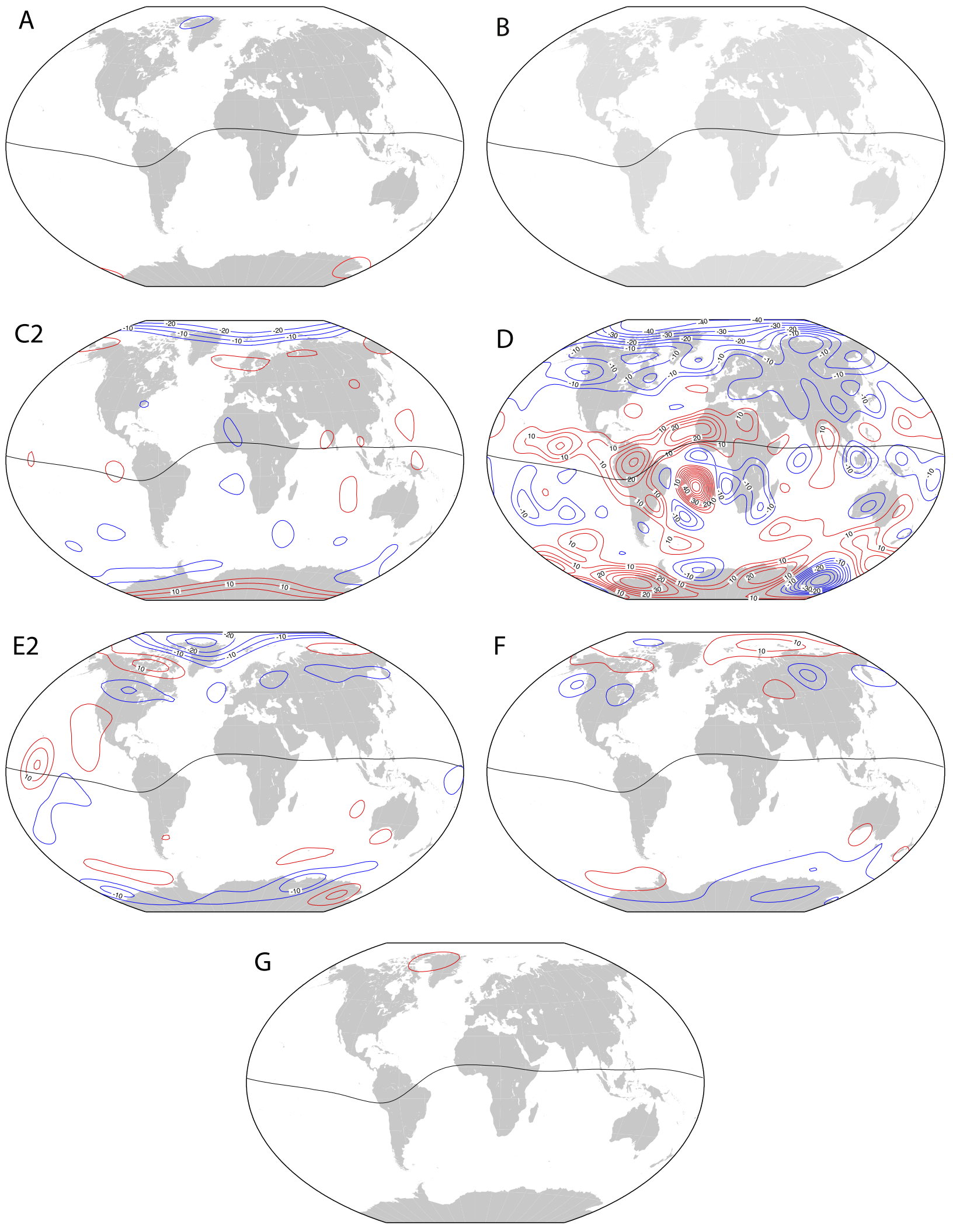

Fig. 3. Difference in the $Z$ component of the magnetic field between each DGRF-2005 candidate model and the mean model $M_{\mathrm{ABG}}$ (i.e. the final DGRF-2005) plotted at Earth's reference radius in Winkel tripel projection. Contours are at intervals of $5 \mathrm{nT}$ with labels every $10 \mathrm{nT}$ when sufficiently large. Red: positive, Blue: negative. The dip equator is shown. 


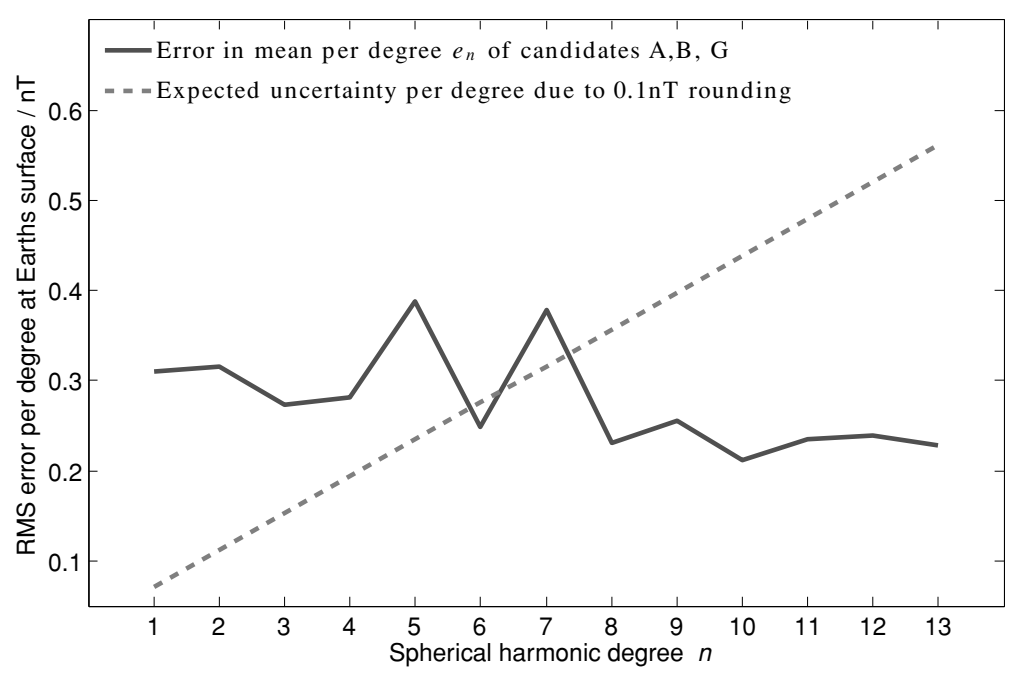

Fig. 4. Standard error $e_{n}$ in the mean per degree defined by (10) and (11), calculated from the mean of DGRF-2005 candidates A, B and G is shown as the solid line. It was assumed that (within a given degree) all the candidates had the same standard deviation. The dashed curve shows the expected uncertainty due to rounding to $0.1 \mathrm{nT}$, given by the expression $0.1 \sqrt{(2 n+1)(n+1) / 12}$. Note that above degree 7 the uncertainty due to rounding is greater than the error in the mean.

relation to $M$ above degree 9 . The degree correlation of candidates $\mathrm{C} 2$, E2 and $\mathrm{F}$ to $M$ above degree 10 is slightly lower than that of A, B and $\mathrm{G}$ which appear similar to each other and close to $M$.

In Fig. 2 coefficient by coefficient analysis of the DGRF2005 candidate models is presented. The plot on the left shows differences ${ }_{i, j} g_{n}^{m}$ as defined in (1) between the candidate models and the arithmetic mean model $M$. The largest differences from $M$ are found to occur for candidate D, with significant deviations also notable for candidates E2, $\mathrm{C} 2$ and $\mathrm{F}$. The deviations associated with candidates A, B and $\mathrm{G}$ are smaller, so that the curves for candidates $\mathrm{A}$ and $\mathrm{B}$ are largely hidden behind those for the other candidates. The right hand plot shows the Huber weights calculated during the determination of robust mean coefficients. Notice that the coefficients of candidate D often receive the lowest weights, particularly for the coefficients associated with the highest harmonics which receive weights as low as 0.4 . Candidates E2, C2 and F also receive low weights for certain coefficients; in particular E2 receives some low weights for coefficients between $n=6$ and $n=9$. Almost all coefficients of candidates $A, B$ and $G$ receive full weights of 1.0 illustrating that they are consistently closer to the robust mean, so are arguably of higher quality.

3.1.3 Spatial analysis of DGRF-2005 candidate models A geographical investigation of the DGRF-2005 candidate models is presented in Fig. 3. This shows the differences between the vertical $(Z)$ component of the candidates and model $M_{\mathrm{ABG}}$ at radius $r=a$. Model $M_{\mathrm{ABG}}$ was chosen as a suitable reference based on the earlier analyses presented in Sections 3.1.1 and 3.1.2.

Studying differences between the candidate models and a reference model in space yields insight into the geographical locations where disparities in the candidates are located. Visual inspection of Fig. 3 reveals that candidate D involves the most striking deviations from $M_{\mathrm{ABG}}$ that are locally as large as $50 \mathrm{nT}$. The differences are scattered over the globe and not confined to any particular geographical location, though the largest discrepancies occur in the polar regions and in the mid-Atlantic. Candidates $\mathrm{C} 2$ and E2 display largest deviations from A, B and G in the polar regions (particularly in the Arctic). Model E2 shows one localized anomalous region in the equatorial Pacific while model $\mathrm{F}$ shows rather minor differences at high latitudes and at mid-latitudes in the northern hemisphere. Candidates A, B and $\mathrm{G}$ exhibit only minor differences to the reference model $M_{\mathrm{ABG}}$ demonstrating once more that they are consistent with each other.

3.1.4 Choice of numerical precision for DGRF-2005 An important analysis for DGRF-2005 was to calculate (using (10) and (11)) the error per degree in the unweighted arithmetic means determined for sets of candidate models. Figure 4 shows the result of such a calculation using candidates $A, B$ and $G$, on the assumption that all the candidates have the same per degree sample deviation $s_{n}$, which is estimated from their scatter about the mean. The solid line shows the resulting error in the mean per degree for model $M_{\mathrm{ABG}}$ which is typically around $0.3 \mathrm{nT}$. The dashed line in Fig. 4 shows the expected uncertainty due to rounding the model coefficients to $0.1 \mathrm{nT}$, given by the expression $0.1 \sqrt{(2 n+1)(n+1) / 12}$ (see, for example, Lowes, 2000). It is observed that the error due to $0.1 \mathrm{nT}$ rounding dominates the error in the mean of candidates A, B and G above degree 7. Given the decision by the task force (see next section) to adopt model $M_{\mathrm{ABG}}$ for the DRGF-2005, this necessitates quoting DGRF-2005 to $0.01 \mathrm{nT}$ rather than $0.1 \mathrm{nT}$ to avoid introducing unnecessary rounding errors. Note that based on internal consistency, the total formal RMS error in the mean model $M_{\mathrm{ABG}}$ (which is DGRF-2005) is remarkably only $1.0 \mathrm{nT}$.

3.1.5 Discussion and summary for DGRF-2005 Based on the tests presented above, candidate D appears consistently different in both the spectral domain (with certain spherical harmonic coefficients apparently 
Table 3. RMS vector field differences ${ }_{i, j} R$ in units of nT between candidate models for IGRF-10 epoch 2005.0, the IGRF-2005 from IGRF-10 and the DGRF-2005 from IGRF-11. Note the symmetry about the diagonal, included as a check on the calculations.

\begin{tabular}{|c|c|c|c|c|c|c|}
\hline$i, j R$ & IGRF-2005-A1 & IGRF-2005-B3 & IGRF-2005-C1 & IGRF-2005-D1 & IGRF-2005 & DGRF-2005 \\
\hline IGRF-2005-A1 & 0.0 & 8.0 & 14.6 & 15.8 & 7.0 & 9.9 \\
\hline IGRF-2005-B3 & 8.0 & 0.0 & 11.4 & 15.6 & 4.6 & 10.9 \\
\hline IGRF-2005-C1 & 14.6 & 11.4 & 0.0 & 20.4 & 8.3 & 18.5 \\
\hline IGRF-2005-D1 & 15.7 & 15.6 & 20.4 & 0.0 & 16.1 & 14.0 \\
\hline IGRF-2005 & 7.0 & 4.6 & 8.3 & 16.1 & 0.0 & 12.0 \\
\hline DGRF-2005 & 9.9 & 10.9 & 18.5 & 14.0 & 12.0 & 0.0 \\
\hline
\end{tabular}

Table 4. Summary of IGRF-2010 candidate models submitted for consideration in IGRF-11.

\begin{tabular}{|c|c|c|c|c|}
\hline \hline \multicolumn{2}{|c|}{ IGRF candidate models for main field epoch 2010 } \\
\hline \hline Team & Model & Organization & Data & Comments (parent model, fwd propagation etc.) \\
\hline \hline A & IGRF-2010-A & $\begin{array}{c}\text { DTU Space / } \\
\text { IPGP / NASA-GSFC }\end{array}$ & $\begin{array}{c}\text { Ørsted, CHAMP, SAC-C } \\
\text { revised observatory monthly means }\end{array}$ & $\begin{array}{c}\text { Based on CHAOS-3 } \alpha \\
\text { evaluated in 2010.0 }\end{array}$ \\
\hline B & IGRF-2010-B & NGDC-NOAA / GFZ & CHAMP 2006.5-2009.7 & $\begin{array}{c}\text { Based on POMME 6: 2nd order Taylor } \\
\text { series SV \& SA used for 2010.0 estimate }\end{array}$ \\
\hline C & IGRF-2010-C2 & BGS & $\begin{array}{c}\text { Ørsted, CHAMP, observatory hourly means } \\
\text { for 01:00-02:00 LT, 1999.0-2009.5 }\end{array}$ & $\begin{array}{c}\text { Revised sub: model evaluated 2009.0 } \\
\text { and linear SV used to predict 2010.0 field. }\end{array}$ \\
\hline D & IGRF-2010-D & IZMIRAN & $\begin{array}{c}\text { CHAMP 2004.0-2009.2 } \\
\text { no data selection }\end{array}$ & $\begin{array}{c}\text { Model at 2009.5 extrapolated } \\
\text { extrapolation to 2010 using NOC1, 2 }\end{array}$ \\
\hline E & IGRF-2010-E & $\begin{array}{c}\text { EOST / LPGN / } \\
\text { LATMOS / IPGP }\end{array}$ & $\begin{array}{c}\text { CHAMP June/July 2009 } \\
\text { to 2010.0 using SV models for 2009, 2010. }\end{array}$ \\
\hline F & IGRF-2010-F & $\begin{array}{c}\text { IPGP / EOST / } \\
\text { / LPGN / LATMOS }\end{array}$ & $\begin{array}{c}\text { CHAMP 2008.5-2009.6 } \\
\text { in quadratic) extrapolated to 2010.0 }\end{array}$ \\
\hline G & IGRF-2010-G & GFZ & $\begin{array}{c}\text { CHAMP 2001-2009.6 } \\
\text { observatory hourly means }\end{array}$ & $\begin{array}{c}\text { Based on GRIMM2 MF and SV } \\
\text { in 2009 extrapolated to 2010.0 }\end{array}$ \\
\hline \hline
\end{tabular}

Table 5. RMS vector field differences ${ }_{i, j} R$ in units of nT between IGRF-2010 candidates and also between them and the arithmetic mean of all candidates $M$ and the weighted mean $M_{w}$ (see text). The bottom row displays the mean of the RMS vector field differences between each candidate model and all other candidate models ${ }_{i} \bar{R}$ from (7) labelled 'Mean Diff'.

\begin{tabular}{|c|r|r|r|r|r|r|r||r|r||r|}
\hline \hline$i, j R$ & $\mathrm{~A}$ & $\mathrm{~B}$ & $\mathrm{C} 2$ & $\mathrm{D}$ & $\mathrm{E}$ & $\mathrm{F}$ & $\mathrm{G}$ & $M^{\prime}$ & $M_{w}$ \\
\hline \hline $\mathrm{A}$ & 0.0 & 6.3 & 10.6 & 14.2 & 14.8 & 8.2 & 8.2 & 6.3 & 6.4 \\
\hline $\mathrm{B}$ & 6.3 & 0.0 & 8.1 & 13.9 & 13.4 & 5.2 & 5.4 & 3.8 & 3.0 \\
\hline $\mathrm{C} 2$ & 10.6 & 8.1 & 0.0 & 16.9 & 11.8 & 10.0 & 8.9 & 7.1 & 6.8 \\
\hline $\mathrm{D}$ & 14.2 & 13.9 & 16.9 & 0.0 & 19.4 & 15.0 & 14.2 & 12.3 & 13.4 \\
\hline $\mathrm{E}$ & 14.8 & 13.4 & 11.8 & 19.4 & 0.0 & 14.0 & 12.4 & 10.9 & 12.0 \\
\hline $\mathrm{F}$ & 8.2 & 5.2 & 10.0 & 15.0 & 14.0 & 0.0 & 6.6 & 5.8 & 5.3 \\
\hline $\mathrm{G}$ & 8.2 & 5.4 & 8.9 & 14.2 & 12.4 & 6.6 & 0.0 & 4.6 & 4.4 \\
\hline \hline Mean Diff & 10.4 & 8.7 & 11.1 & 15.6 & 14.3 & 9.8 & 9.3 & 7.3 & 7.3 \\
\hline \hline
\end{tabular}

anomalous—-see Fig. 2) as well as in physical space where global problems are observed. In addition candidates E2, $\mathrm{C} 2$ and to lesser extent $\mathrm{F}$ were observed to have some problems, particularly at high degrees in the spectral domain and at high latitudes in space. In contrast candidates A, B and G were very similar despite being derived using different data selection criteria and using different modelling procedures. The task force therefore voted that DGRF-2005 be derived from a simple arithmetic mean of candidates $A, B$ and $G$ (i.e. model $M_{\mathrm{ABG}}$ as discussed above).

\subsection{Retrospective analysis of IGRF-10 MF candidate models for epoch 2005}

Having established a new DGRF for epoch 2005 it is possible to carry out an assessment of the quality of the candidate models that contributed to the IGRF-10 provisional model for epoch 2005. Table 3 presents the RMS vector field differences $i, j R$ between the various candidate models, the IGRF-2005 model (from IGRF-10) and the DGRF2005 model (from IGRF-11). The naming convention for the candidates is that used by Maus et al. (2005). Candidate A1 was a model from DSRI/NASA/Newcastle, Candidate B3 was a model from NGDC/GFZ, Candidate C1 was a model from BGS and Candidate D1 was a candidate from IZMIRAN. Candidate A1 agrees most closely with DGRF2005 with a global RMS vector field difference of $9.9 \mathrm{nT}$ followed closely by B3 which differs by $10.9 \mathrm{nT}$. Candidate D1 does a little worse with a difference $14.0 \mathrm{nT}$ and candidate $\mathrm{C} 1$ is furthest from DGRF-2005 with an RMS vector field difference of $18.5 \mathrm{nT}$, almost twice that of candidate A1. The IGRF-2005 (which was the arithmetic mean 


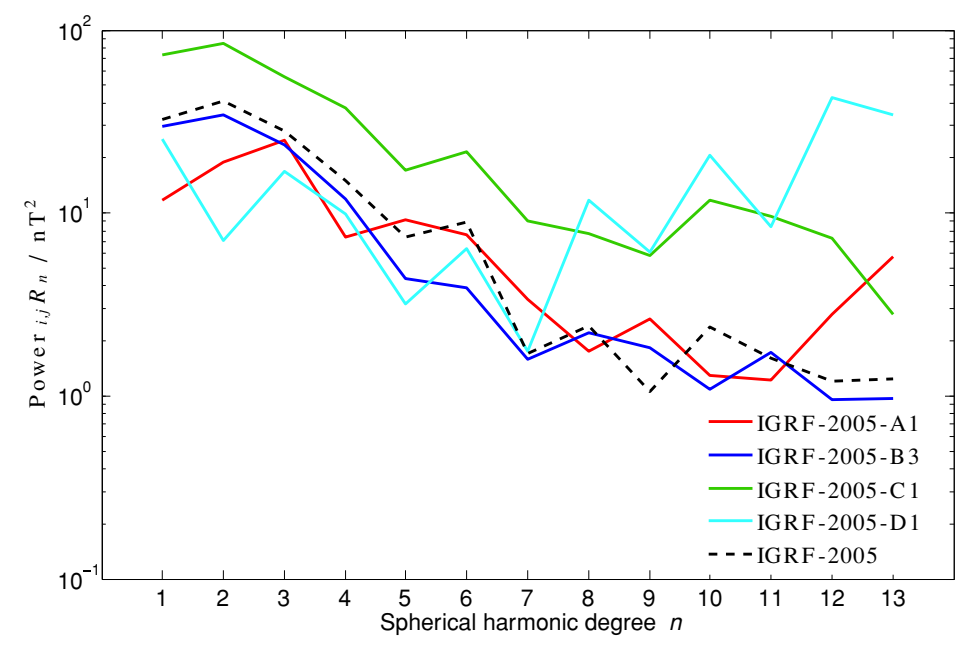

Fig. 5. Lowes-Mauersberger spectra of the vector field differences ${ }_{i, j} R_{n}$ between DGRF-2005 and the candidate models considered for IGRF-10 epoch 2005.0.
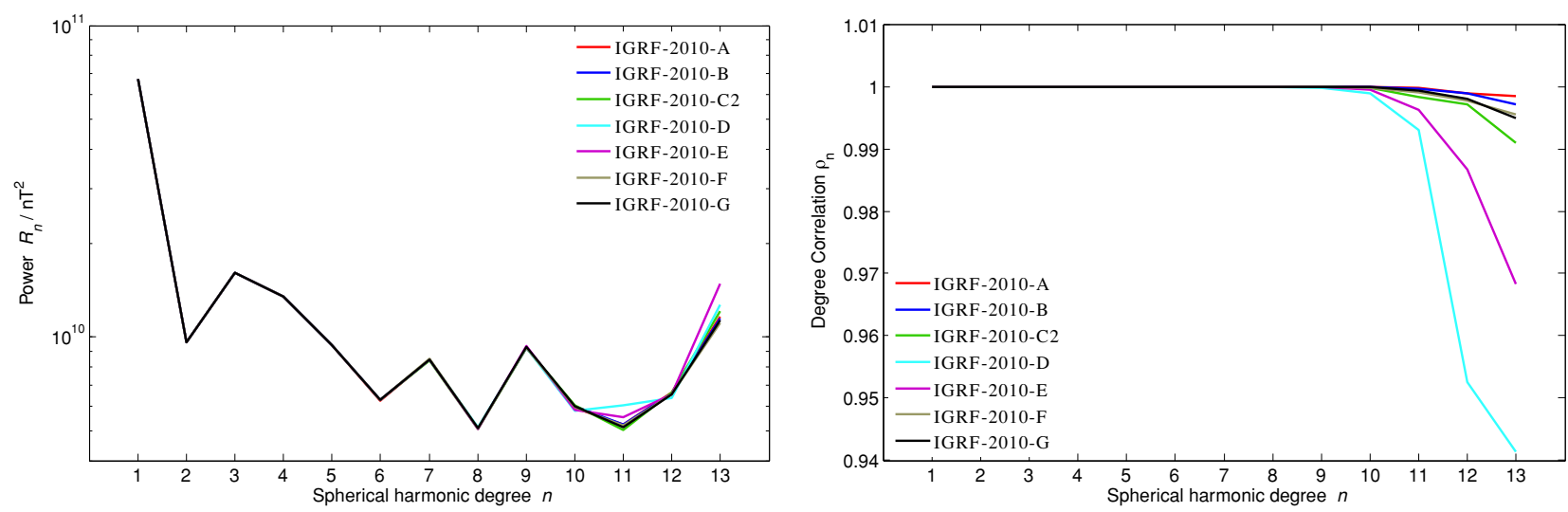

Fig. 6. Lowes-Mauersberger power spectra ${ }_{i, 0} R_{n}$ from (3) of IGRF-2010 candidate models at radius $3480 \mathrm{~km}$ (core-mantle boundary) (left) and degree correlation $_{i, M} \rho_{n}$ (9) of IGRF-2010 candidate models with the arithmetic mean model $M$ (right).
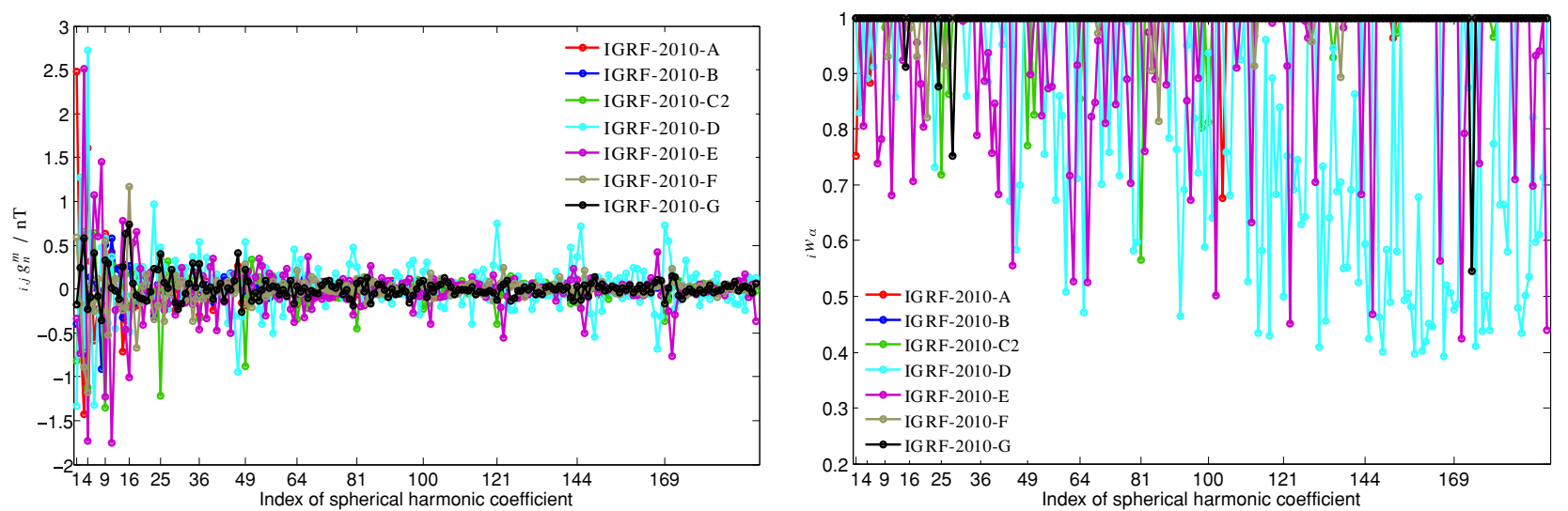

Fig. 7. Left plot shows differences $i_{i, j} g_{n}^{m}$ as defined in (1) between IGRF-2010 candidate models and the arithmetic mean Model $M$ as a function of the index of the spherical harmonic coefficient (running from $g_{1}^{0}, g_{1}^{1}, h_{1}^{1}, g_{2}^{0}, h_{1}^{1}$ etc indexed 1, 2, 3, 4, 5, etc). Right plot shows Huber robust weighting factors ${ }_{i} w_{\alpha}$ for the candidate models for IGRF-2010 (1.0 full weight, 0.0 zero weight) also as a function of the index of the spherical harmonic coefficient.

of candidates A1, B3 and C1) differed from DGRF-2005 by $12.0 \mathrm{nT}$.

In Fig. 5 the difference in power per degree between the IGRF-10 candidates and DGRF-2005 $\left(i, j, R_{n}\right)$ are presented.
The mean square vector field difference per degree between the final IGRF-2005 (the arithmetic mean of A1, B3 and C1) and DGRF-2005 is shown as the black dashed line. It appears that the problems with candidate D1 are pre- 
dominantly at high degree $(n>7)$; it is better than most other candidates at the lower degrees. Candidate $\mathrm{C} 1$ was further from DGRF-2005 than all the other candidates at low degrees 1-7 suggesting some systematic problem with this model. It is also noticeable that candidate A1 did better than the other candidates for the dipole $(n=1)$ terms while candidate B3 performed best at high degrees, especially $n=12,13$.

\subsection{Analysis of IGRF-11 MF candidate models for epoch 2010}

Having completed the analysis of MF models for epoch 2005.0 we now move on to consider epoch 2010.0. Table 4 summarizes the candidate models submitted for IGRF-2010. Note that model C2 was a resubmission by BGS who withdrew their initial candidate. Further details are again given in the papers in this special issue focusing on the various candidate models, and their descriptions are available online at http://www. ngdc.noaa.gov/IAGA/vmod/candidatemodels.html. Models for epoch 2010.0 were submitted in October 2009; teams therefore faced the additional challenge of how to propagate their estimates forward to 2010.0; this was not an issue faced when deriving retrospective models for epoch 2005.0. A brief indication of the method used to propagate to epoch 2010.0 is provided in the final column of Table 4. Larger differences in the candidate models are expected due to this additional complication; it the IGRF-11 model for epoch 2010.0 is therefore only provisional and will be updated to a DGRF in 2014 during the IGRF-12 process.

3.3.1 RMS vector field differences for IGRF-2010 candidate models Table 5 displays the RMS vector field differences $_{i, j} R$ between the IGRF-11 candidates for epoch 2010.0 and also between the candidates and the arithmetic mean model $M$ and a weighted mean model $M_{w} . M_{w}$ is reported here because it was important in the final voting process; it consists of candidates A, B, C2, F and G having weight 1.0 and candidates $\mathrm{D}, \mathrm{E}$ having weight 0.25 (in addition coefficients $g_{1}^{0}$ and $h_{1}^{1}$ of candidate A were disregarded following a vote by the task force). The bottom row of Table 5 shows ${ }_{i} \bar{R}$, the mean of the differences ${ }_{i, j} R$ (excluding the zero value for the difference between candidates and themselves-see (7)).

As anticipated, the differences between the IGRF-2010 candidates are larger than between the DGRF-2005 candidates, with the mean of the differences between the candidates and the mean model (i.e. the mean of ${ }_{i, M} R$ ) being $7.3 \mathrm{nT}$ here for epoch 2010.0 compared to $4.9 \mathrm{nT}$ for epoch 2005.0. Candidates D and E display the largest differences from the other candidates and to the mean models $M$ and $M_{w}$. Candidate $\mathrm{B}$ is most similar to $M$ and it also agrees reasonably closely with candidates $\mathrm{F}$ and $\mathrm{G}$ (differences less than $5.5 \mathrm{nT}$ ) and slightly less well with candidates A and C2 (differences of less than $8.5 \mathrm{nT}$ ).

3.3.2 Spectral analysis of IGRF-2010 candidate models In Fig. 6 (left) we plot the Lowes-Mauersberger spectra $_{i, 0} R_{n}$ from (3) of the IGRF-2010 candidates at the core-mantle boundary. Candidates E and D have noticeably higher power in degrees 11 and 13 suggesting that they may have difficulties with noise being mapped into some model coefficients at high degree.
Figure 6 (right) shows the degree correlation per degree $i, M \rho_{n}$ from (9) between the candidates and the arithmetic mean model $M$. Candidates $\mathrm{E}$ and especially $\mathrm{D}$ show the largest differences above degree 10; candidates $\mathrm{C} 2, \mathrm{~F}$ and $\mathrm{G}$ show smaller deviations from $M$ while candidates A and B are closest to $M$.

In Fig. 7 the left hand plot presents the coefficient by coefficient differences ${ }_{i, j} g_{n}^{m}$ as defined in (1) between the IGRF-2010 candidates and the mean model $M$. It is apparent that there are some systematic problems. Candidate A possesses particularly large differences from $M$ in coefficients $g_{1}^{0}$ and $h_{1}^{1}$. Candidate D displays many remarkable differences from $M$ in the $h_{n}^{n}$ sectoral harmonics while candidate $\mathrm{E}$ shows anomalous $h_{n}^{1}$ coefficients, particularly at degrees $n=11-13$. Candidate $\mathrm{C} 2$ shows differences from $M$ predominantly in the $g_{n}^{0}$ terms, most noticeably in degrees $n=3-9$. The right hand plot in Fig. 7 displays the Huber weights as a function of the index of the spherical harmonic coefficient. It shows how the robust weighting scheme would in this circumstance strongly down-weight many (but not all) of the coefficients of candidate $D$ at $n>10$, as well as many of the $h_{n}^{1}$ coefficients of candidate E. The lowest Huber weight for the important $g_{1}^{0}$ axial dipole coefficient is allocated to candidate A. Aside from this exception candidates $\mathrm{A}, \mathrm{B}, \mathrm{C} 2, \mathrm{~F}$ and $\mathrm{G}$ receive Huber weighting factors close to 1 for the majority of their coefficients.

3.3.3 Spatial analysis of IGRF-2010 candidate models In Fig. 8 we plot at Earth's surface the differences between the $Z$ component of the IGRF-2010 candidate models and the weighted mean model $M_{w}$ in which candidates D and $\mathrm{E}$ are weighted by a factor 0.25 and the $g_{1}^{0}$ and $h_{1}^{1}$ coefficients of candidate A are discarded. The largest discrepancies are observed for candidates D and E. Candidate D displays major differences from $M_{w}$ along the dip equator, and in the high latitude Arctic region where differences as large as $50 \mathrm{nT}$ are evident. Candidate $\mathrm{E}$ also displays prominent deviations from $M_{w}$ in the Arctic region, but predominantly of the opposite sign to those of candidate $\mathrm{D}$; in addition it possesses low latitude anomalies linked to its anomalous sectoral harmonics. For both candidates E and D the deviations are globally distributed rather than localized. Candidate $\mathrm{C} 2$ has its largest differences from the other models in the polar regions. Candidates A, B, F, and G show more minor deviations from $M_{w}$, the differences being largest in the polar regions in all cases. The analysis of the IGRF-2010 candidate models in geographical space highlights that the most serious differences in the candidate models occur in the polar regions and to a lesser extent along the dip equator. Future efforts towards improved field models will require better models of external and induced fields in these regions.

3.3.4 Discussion and summary for IGRF-2010 The evaluations of the IGRF-2010 candidates presented above suggest that candidates D and E have some problems, particularly at spherical harmonic degree greater than 10 . Consequently the task force voted to allocate these candidates weight 0.25 while candidates A, B, C2, F, G were allocated weight 1.0 in the determination of the new IGRF-11 model for epoch 2010. In addition the task force voted to disre- 

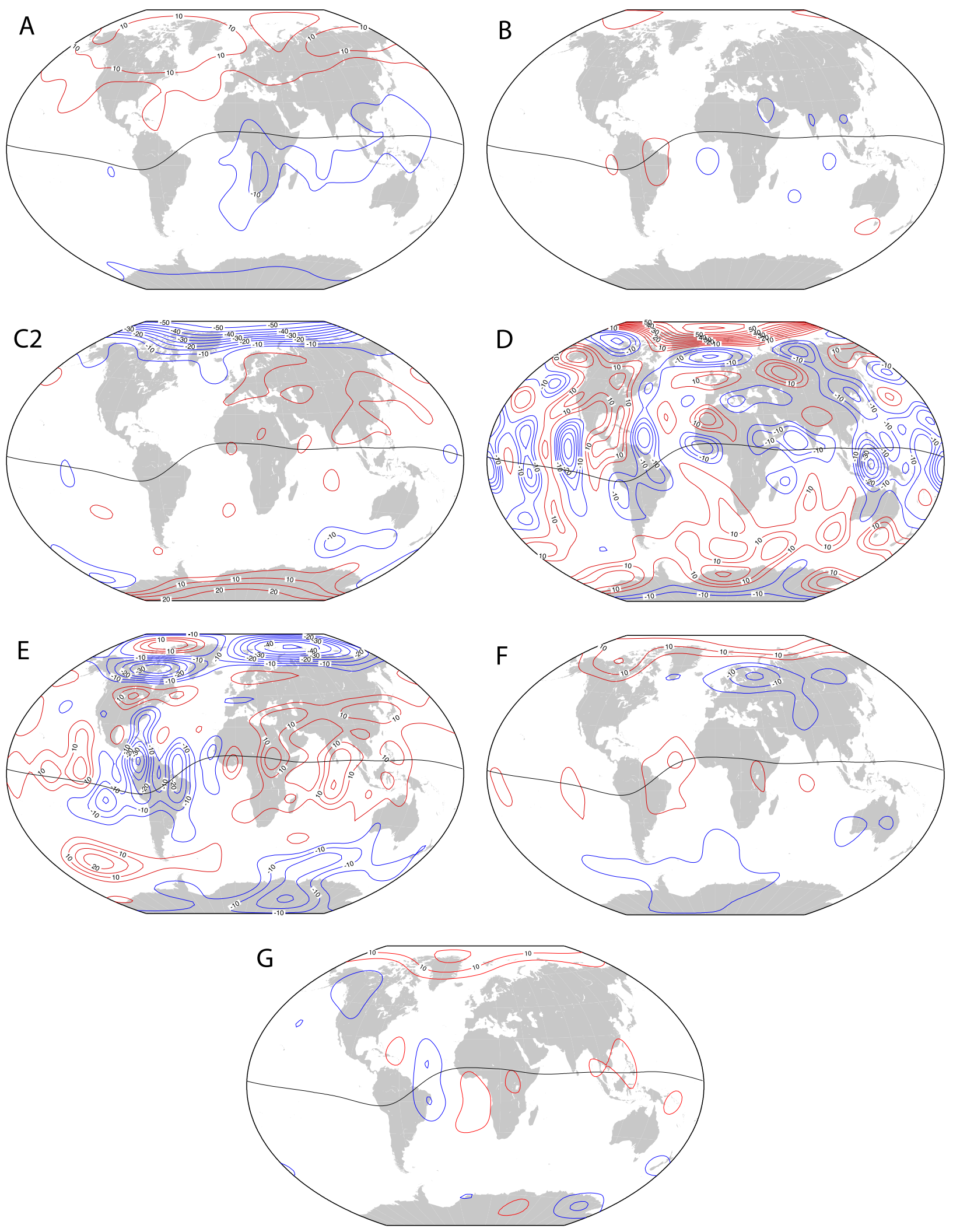

Fig. 8. Difference between the $Z$ component of the magnetic field of IGRF- 2010 candidate models and the weighted mean model $M_{w}$ plotted at Earth's reference radius in Winkel tripel projection. Contours are at intervals of $5 \mathrm{nT}$ with labels every $10 \mathrm{nT}$ when sufficiently large. Red: positive, Blue: negative. The dip equator is shown. 
Table 6. RMS vector field differences ${ }_{i, j} R$ in units of $\mathrm{nT} / \mathrm{yr}$ between SV candidate models from IGRF-10 for epoch 2007.5 , their weighted mean SV-2007.5-G10 and the mean SV between 2005 and 2010 as determined from IGRF-11, using DGRF-2005 and IGRF-2010, SV-2007.5-G11. Note the symmetry about the diagonal, again included as a check on the calculations.

\begin{tabular}{|c|c|c|c|c|c|c|c|}
\hline$i, j R$ & SV-2007.5-A3 & SV-2007.5-B1 & SV-2007.5-B2 & SV-2007.5-C1 & SV-2007.5-D1 & SV-2007.5-G10 & SV-2007.5-G11 \\
\hline SV-2007.5-A3 & 0.0 & 11.1 & 6.7 & 11.8 & 16.9 & 6.0 & 21.9 \\
\hline SV-2007.5-B1 & 11.1 & 0.0 & 12.2 & 17.4 & 19.5 & 11.3 & 21.3 \\
\hline SV-2007.5-B2 & 6.7 & 12.2 & 0.0 & 10.3 & 16.6 & 5.9 & 23.8 \\
\hline SV-2007.5-C1 & 11.8 & 17.4 & 10.3 & 0.0 & 19.1 & 9.4 & 28.4 \\
\hline SV-2007.5-D1 & 16.9 & 19.5 & 16.6 & 19.1 & 0.0 & 12.6 & 20.3 \\
\hline SV-2007.5-G10 & 6.0 & 11.3 & 5.9 & 9.4 & 12.6 & 0.0 & 21.5 \\
\hline SV-2007.5-G11 & 21.9 & 21.3 & 23.8 & 28.4 & 20.3 & 21.5 & 0.0 \\
\hline
\end{tabular}

Table 7. Summary of SV-2010-2015 candidate models submitted to IGRF-11.

\begin{tabular}{|c|c|c|c|c|}
\hline \multicolumn{5}{|c|}{ Predictive SV candidate models for epoch 2010-2015 } \\
\hline Team & Model & Organization & Data & Comments (parent model etc.) \\
\hline A & SV-2010-2015-A & $\begin{array}{c}\text { DTU Space / } \\
\text { IPGP / NASA-GSFC }\end{array}$ & $\begin{array}{c}\text { Ørsted, CHAMP, SAC-C } \\
\text { revised observatory monthly means }\end{array}$ & $\begin{array}{l}\text { Based on CHAOS-3 } \alpha \\
\text { SV at } 2010.0\end{array}$ \\
\hline B & SV-2010-2105-B & NGDC-NOAA / GFZ & CHAMP 2006.5-2009.7 & $\begin{array}{c}\text { Based on POMME 6: 2nd order Taylor } \\
\text { SV at 2009.7 used. }\end{array}$ \\
\hline $\mathrm{C}$ & SV-2010-2015-C2 & BGS & $\begin{array}{c}\text { Ørsted, CHAMP and } \\
\text { observatory hourly means }\end{array}$ & $\begin{array}{l}\text { Revised sub: Av. SV 2005.0-2009.0 } \\
\text { from parent model used }\end{array}$ \\
\hline $\mathrm{D}$ & SV-2010-2105-D & IZMIRAN & CHAMP 2004.0-2009.25 & Based on linear NOC extrapolated \\
\hline $\mathrm{E}$ & SV-2010-2015-E2 & $\begin{array}{l}\text { EOST / LPGN / } \\
\text { LATMOS / IPGP }\end{array}$ & $\begin{array}{l}\text { Observatory hourly mean used } \\
\text { to derive monthly means } 1980-1998\end{array}$ & $\begin{array}{l}\text { Extrap. gives 1st diff of ann. means } \\
\text { 1981-2015: SV models is averaged over last } 6 \text { yrs }\end{array}$ \\
\hline $\mathrm{F}$ & SV-2010-2015-F & $\begin{array}{c}\text { IPGP / EOST / } \\
\text { / LPGN / LATMOS }\end{array}$ & CHAMP 2008.5-2009.6 & $\begin{array}{l}\text { 2nd order Taylor series (to } n=5) \text { : } \\
\text { used slope at 2009.0. }\end{array}$ \\
\hline G & SV-2010-2015-G & GFZ & $\begin{array}{c}\text { CHAMP 2001-2009.6 } \\
\text { Observatory hourly means }\end{array}$ & $\begin{array}{c}\text { Based on GRIMM2: linear fit. } \\
\text { SV 2001.0-2009.5, extrapolated to } 2012.5 \text {. }\end{array}$ \\
\hline $\mathrm{H}$ & SV-2010-2015-H & $\begin{array}{c}\text { NASA GSFC / } \\
\text { UMBC / Univ. Liverpool }\end{array}$ & & $\begin{array}{l}\text { Geodynamo simulation, with assimilation } \\
\text { from CALS7K.2, gufm } 1, \text { CM4, CHAOS- } 2 \mathrm{~s}\end{array}$ \\
\hline
\end{tabular}

Table 8. RMS vector field differences ${ }_{i, j} R$ in units nT/yr between SV-2010-2015 candidate models and between these and the mean model $M$ and the weighted mean model $M_{w}$ in the rightmost columns. The final row labelled 'Mean Diff' is the mean ${ }_{i} \bar{R}$ of the $i, j=$ for each candidate or mean model.

\begin{tabular}{|c|r|r|r|r|r|r|r|r|r||r|}
\hline \hline$i, j R$ & \multicolumn{1}{|c|}{$\mathrm{A}$} & $\mathrm{B}$ & \multicolumn{1}{c|}{$\mathrm{C} 2$} & $\mathrm{D}$ & $\mathrm{E}$ & $\mathrm{F}$ & $\mathrm{G}$ & $\mathrm{H}$ & $M^{\prime}$ & $M_{w}$ \\
\hline \hline $\mathrm{A}$ & 0.0 & 10.0 & 20.2 & 15.9 & 22.2 & 11.4 & 21.0 & 18.1 & 12.8 & 13.8 \\
\hline $\mathrm{B}$ & 10.0 & 0.0 & 15.4 & 10.2 & 18.3 & 5.1 & 18.1 & 12.5 & 7.4 & 7.8 \\
\hline $\mathrm{C} 2$ & 20.2 & 15.4 & 0.0 & 8.0 & 11.0 & 11.4 & 24.2 & 6.5 & 9.7 & 8.6 \\
\hline $\mathrm{D}$ & 15.9 & 10.2 & 8.0 & 0.0 & 12.7 & 6.7 & 18.2 & 4.7 & 4.1 & 3.5 \\
\hline $\mathrm{E}$ & 22.2 & 18.3 & 11.0 & 12.7 & 0.0 & 15.3 & 26.3 & 11.6 & 12.9 & 12.6 \\
\hline $\mathrm{F}$ & 11.4 & 5.1 & 11.4 & 6.7 & 15.3 & 0.0 & 18.1 & 9.0 & 4.1 & 4.3 \\
\hline $\mathrm{G}$ & 21.0 & 18.1 & 24.2 & 18.2 & 26.3 & 18.1 & 0.0 & 20.7 & 16.9 & 17.8 \\
\hline $\mathrm{H}$ & 18.1 & 12.5 & 6.5 & 4.7 & 11.6 & 9.0 & 20.7 & 0.0 & 6.6 & 5.7 \\
\hline Mean Diff & 17.0 & 12.8 & 13.8 & 10.9 & 16.8 & 11.0 & 21.0 & 11.9 & 9.3 & 9.3 \\
\hline \hline
\end{tabular}

gard coefficients $g_{1}^{0}$ and $h_{1}^{1}$ from candidate A since these were thought to be suspect. Subsequent analysis has shown that a model that includes more recent data but is otherwise similar to the parent model for candidate A results in values of $g_{1}^{0}$ and $h_{1}^{1}$ that are in much better agreement with model $M$ (Olsen et al., 2010). The final IGRF-2010 was therefore fixed to be the model discussed above as $M_{w}$.

\section{Evaluation of Predictive SV Candidate Models 4.1 Retrospective analysis of IGRF-10 SV-2005-2010 candidate models}

With the evaluations of the main field candidates for epoch 2005.0 and 2010.0 complete we now move on to consider evaluations of predictive SV models. First we present a retrospective analysis of the predictive average SV-2005-2010 candidates (with central epoch 2007.5) used in IGRF-10. We treat as a reference SV model IGRF-2010 minus DGRF-2005 divided by 5 years - this provides the required coefficients in $\mathrm{nT} / \mathrm{yr}$ centered on epoch 2007.5. 


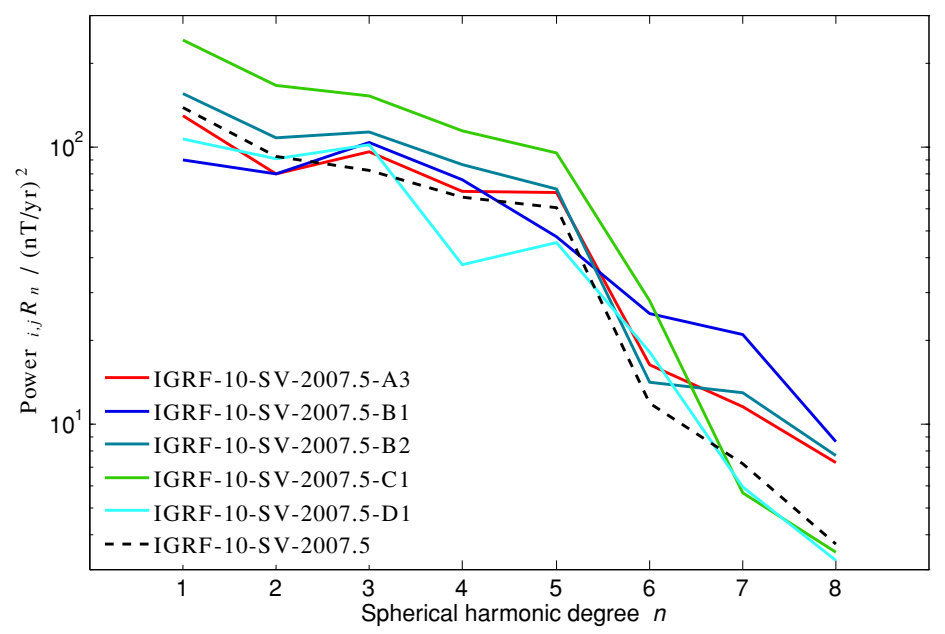

Fig. 9. Lowes-Mauersberger spectra of the vector field differences $i, j R_{n}$ between the IGRF-11 average SV over the interval 2005 to 2010 (IGRF-2010 minus DGRF-2005 divided by 5, referred to as SV-2007.5-G11 in the text) and the SV candidate models considered for IGRF-10 epoch at 2007.5.
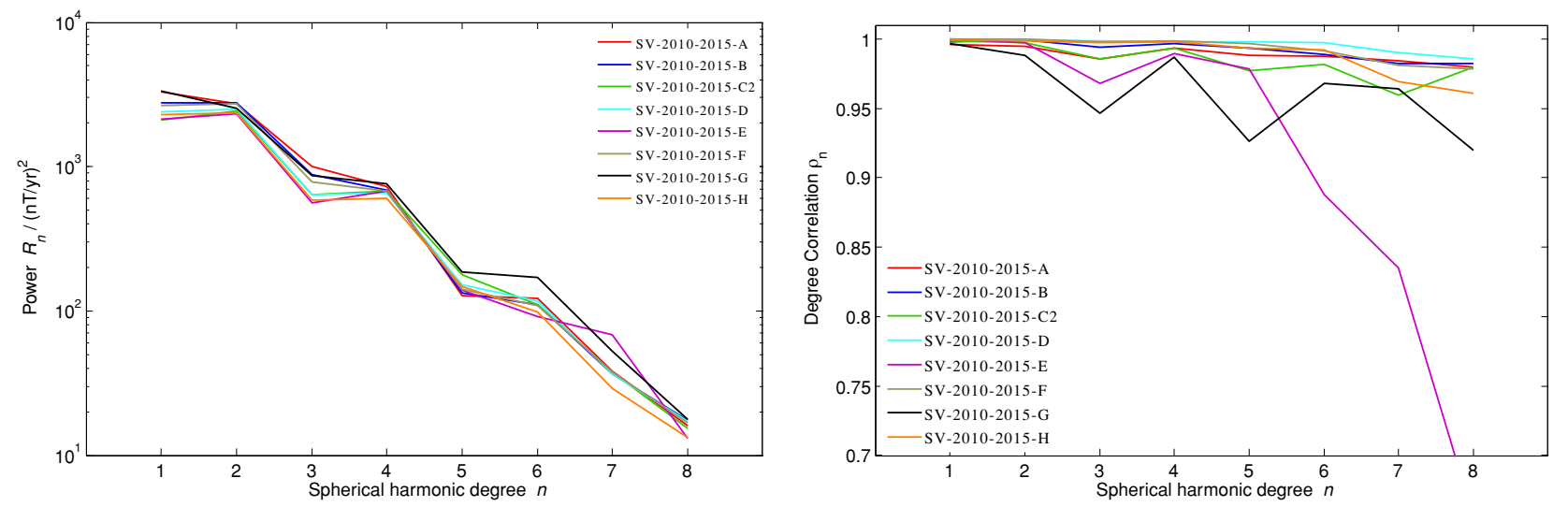

Fig. 10. Lowes-Mauersberger spectra ${ }_{i, 0} R_{n}$ in units $(\mathrm{nT} / \mathrm{yr})^{2}$ from (3) of SV-2010-2015 candidates at Earth's surface (left) and the degree correlation $i, M \rho_{n}$ from (9) between the candidate models and a mean model $M$ (right).
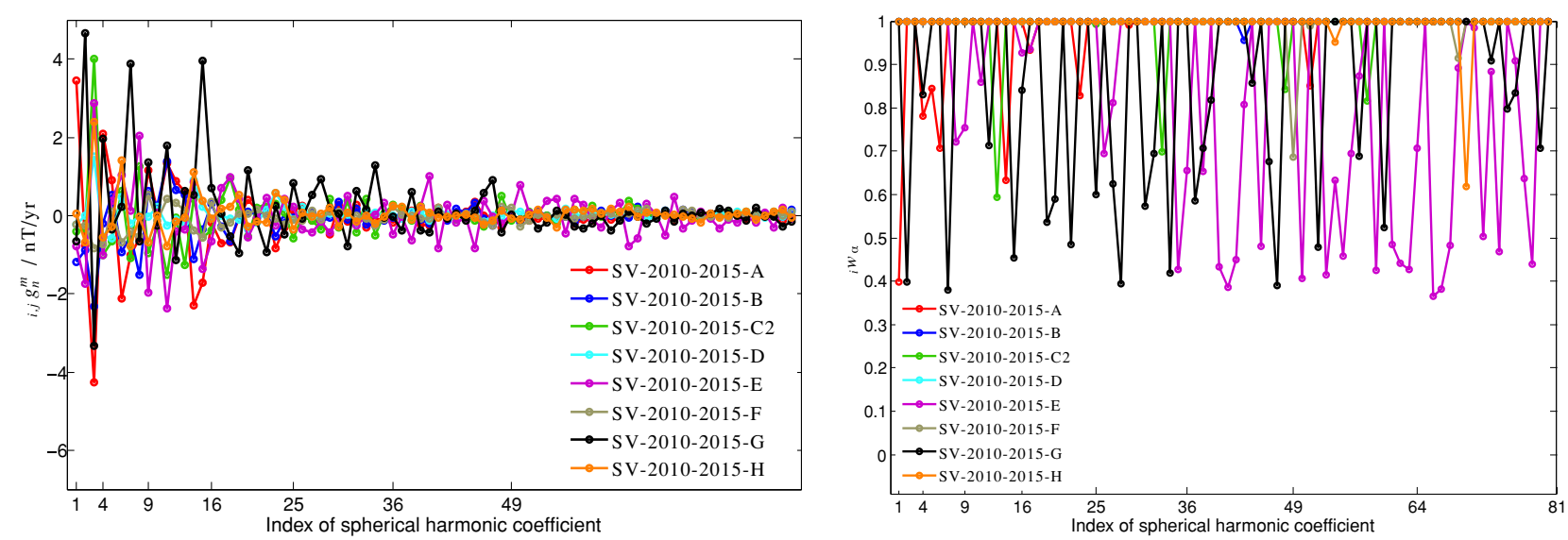

Fig. 11. Left plot shows differences ${ }_{i, j} g_{n}^{m}$ in units nT/yr defined in (1) between SV 2010-2015 candidate models and the mean model $M$ as a function of the index of the spherical harmonic coefficient (running from $g_{1}^{0}, g_{1}^{1}, h_{1}^{1}, g_{2}^{0}, h_{1}^{1}$ etc indexed 1, 2, 3, 4, 5, etc). Right plot shows Huber robust weighting factor ${ }_{i} w_{\alpha}$ (1.0 full weight, 0.0 zero weight) also as a function of the index of the spherical harmonic coefficient.

We refer to this model in the following discussion as SV2007.5-G11. The predictive SV from IGRF-10 (a weighted mean of IGRF-10 candidates A3, C1 and D1 with weight 1.0 , and $\mathrm{B} 1$ and $\mathrm{B} 2$ with weight 0.5 ) is referred to in the following as SV-2007.5-G10. Candidate A1 was from DSRI/NASA/Newcastle, candidates B1, B2 were models from NGDC/GFZ, candidate $\mathrm{C} 1$ was a model from BGS and candidate D1 was produced by IZMIRAN. For further 
details on the IGRF-10 candidate models readers should consult Maus et al. (2005).

In Table 6 we report the RMS vector field differences ${ }_{i, j} R$ between the IGRF-10 SV candidate models, their weighted mean SV-2007.5-G10, and the model derived from IGRF11, SV-2007.5-G11. Compared to the SV derived retrospectively from the IGRF-11 MF models (SV-2007.5-G11), the IGRF-10 candidate model D1 was found to perform best with an RMS difference of $20.3 \mathrm{nT} / \mathrm{yr}$. Candidates A3 and B1 did almost as well with differences of 21-22 nT/yr. Candidate B2 performed slightly less well with a difference of almost $24 \mathrm{nT} / \mathrm{yr}$ while candidate $\mathrm{C} 1$ performed worst with a difference of $28.4 \mathrm{nT} / \mathrm{yr}$. In comparison, the IGRF-10 SV prediction differed from the IGRF-11 model by $21.5 \mathrm{nT} / \mathrm{yr}$. Interestingly Beggan and Whaler (2010), also in this issue, demonstrate that by using a steady, tangentially geostrophic, core flow they are able to derive a predictive SV model that performs slightly better than any of the candidate models for IGRF-10, with a RMS vector field difference of $\sim 17 \mathrm{nT} / \mathrm{yr}$.

In Fig. 9 the power spectra of the mean square vector field differences per degree between the candidates and also SV-2007.5-G10 compared to SV-2007.5-G11 are presented. The difference between SV-2007.5-G10 and SV2007.5-G11 is shown as the black dashed line. Candidate $\mathrm{C} 1$ is found to have the largest differences at all degrees less than 6 while candidate B2 performs most poorly at degrees 7 and 8. Candidates A3, B1 and B2 involved extrapolation via quadratic terms out to 2007.5 and consequently had higher power at high degrees; the simpler linear models $\mathrm{C} 1$ and D1 are found in this case to perform better at high degree suggesting that extrapolation using quadratic terms was not beneficial.

\subsection{Analysis of IGRF-11 SV-2010-2015 candidate models}

The final evaluation carried out was that of candidates submitted for the IGRF-11 average predictive SV for the interval 2010.0-2015.0. Note that in this section $g_{n}^{m}$ and $h_{n}^{m}$ refer to the predicted average annual rate of change in the coefficients between epochs 2010.0 and 2015.0 in units of nT/yr. SV candidates were sought only to degree 8 , although test models to higher degree were also submitted by some teams and are available for future evaluations from the webpage http://www. ngdc.noaa.gov/IAGA/vmod/candidatemodels.html. These test models should help to determine whether it is worthwhile to extend the truncation level for predictive SV in IGRF-12 (for further discussion of this point see Silva et al. (2010), this issue). Eight teams submitted SV-20102015 candidates, the same teams that submitted candidates for DGRF-2005 and IGRF-2010 and in addition a team, referred to as team H, led by Weijia Kuang at NASA-GSFC (Kuang et al., 2010). The latter team for the first time in the history of IGRF used assimilation of a retrospective field models including (CHAOS-2s) into a geodynamo simulation based on an approximation of core dynamics in order to obtain a SV forecast for the upcoming 5 years. Details of the teams submitting SV candidates are collected for reference in Table 7.
4.2.1 RMS vector field differences for SV-2010-2015 candidate models Analysis begins as before with a compilation of RMS vector field differences ${ }_{i, j} R$ in Table 8 between the candidates and an arithmetic mean model $M$ and a weighted mean model $M_{w}$ (the latter in this case consists of candidates B, C2, D, F, H with weight 1.0 and candidates A, E and G with weight 0.5 with coefficients $g_{1}^{0}$ and $h_{1}^{1}$ of A discarded again following a vote by the task force). In comparison to the earlier analyses of the MF models there is much more spread in the predictions of the SV candidate models with the mean of ${ }_{i, j} R$ between candidates being $14.4 \mathrm{nT} / \mathrm{yr}$. For IGRF-2010 it was evident that a group of models (A, B, C2, F and G) were consistently similar, so a strong down-weighting (by a factor of 0.25 ) of the remaining models was agreed on by the task force. In contrast for SV-2010-2015 since there was a larger scatter amongst the candidate models and it was less clear that particular candidates should be strongly down-weighted. The task force thus decided on a less severe weighting factor of 0.5 in this case. Candidates D and $\mathrm{F}$ are now the closest to the mean model $M$, followed by candidates $\mathrm{H}, \mathrm{B}$ and $\mathrm{C} 2$, then candidates $\mathrm{A}$ and $\mathrm{E}$, with candidate $\mathrm{G}$ most different from $M$.

4.2.2 Spectral analysis of SV-2010-2015 candidate models Given the spread in the candidate models it is instructive to consider the Lowes-Mauersberger spectra (3) of the SV candidates at Earth's surface in Fig. 10 (left), rather than at the core-mantle boundary as was the case for the MF models. There appears to be no obvious way to choose between the candidates; they are widely spread at all degrees rather than being largely consistent with a few anomalous outliers. Candidate $\mathrm{G}$ contains noticeably more power in degree 6 while candidate $E$ has a very different spectral slope for degrees 5 to 8 (with degree 7 appearing anomalously high). The degree correlation ${ }_{i, M} \rho_{n}$ from (9) between the candidate models and mean model $M$ as shown in Fig. 10 (right) illustrates that candidate E possesses a lower correlation to the mean model above degree 5 , while candidate $\mathrm{G}$ is also noticeably different in degrees 3, 5 and 8. Candidate $\mathrm{C} 2$ also has marginally lower degree correlations to $M$ than the remaining models, but it is less obviously different than candidates $\mathrm{E}$ and $\mathrm{G}$.

In Fig. 11 the left hand plot presents the coefficient by coefficient differences defined in (1), between the candidate models and model $M$ while the right hand plot presents the Huber weights allocated by the robust weighting procedure. Candidate $\mathrm{E}$ is consistently allocated Huber weights as low as 0.4 for many coefficients above degree 6 , while candidate $\mathrm{G}$ possesses some noticeably anomalous coefficients even at low degree (this is also apparent in the plot of ${ }_{i, M} \rho_{n}$ in Fig. 10).

4.2.3 Spatial analysis of SV-2010-2015 candidate models In Fig. 12 the differences in the vertical $(Z)$ component of SV at the Earth's surface between $M_{w}$ and the SV-2010-2015 candidates is presented. The largest difference from the weighted mean is observed for candidates $G$ and $E$. Candidate $G$ predicts a large negative change in the $Z$ to the west of Australia that is not present in the other candidates; this feature has a maximum amplitude that is more than $55 \mathrm{nT} / \mathrm{yr}$ different from $M_{w}$. The majority of the differences for candidate $\mathrm{G}$ occur at low latitudes. Candi- 

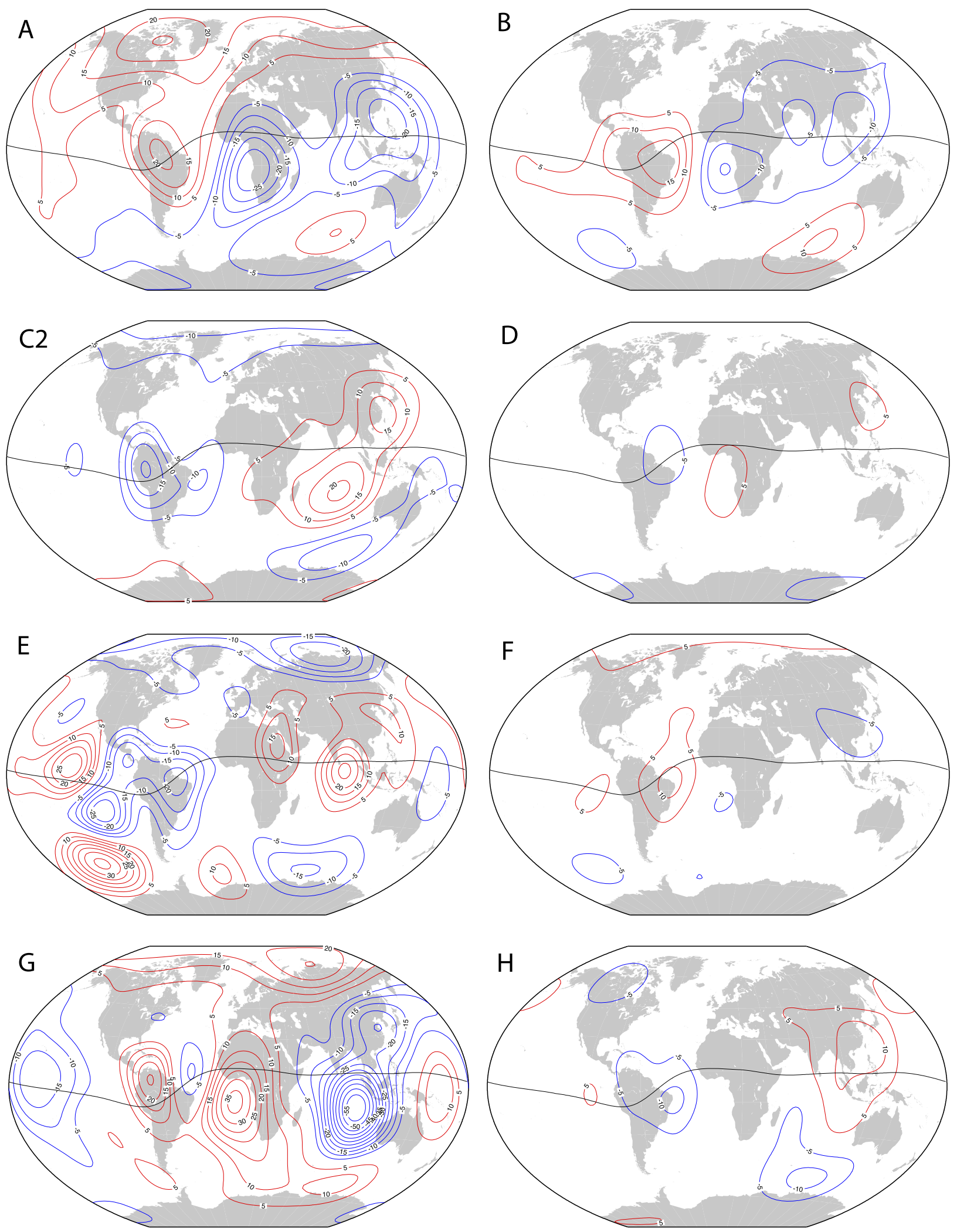

Fig. 12. Difference in the $Z$ component of the magnetic field between SV-2010-2015 candidate models and the weighted mean model $M_{w}$ plotted at Earth's reference radius in Winkel tripel projection. Contours are at intervals of $5 \mathrm{nT} / \mathrm{yr}$; all are labelled. Red: positive, Blue: negative. The dip equator is shown. 


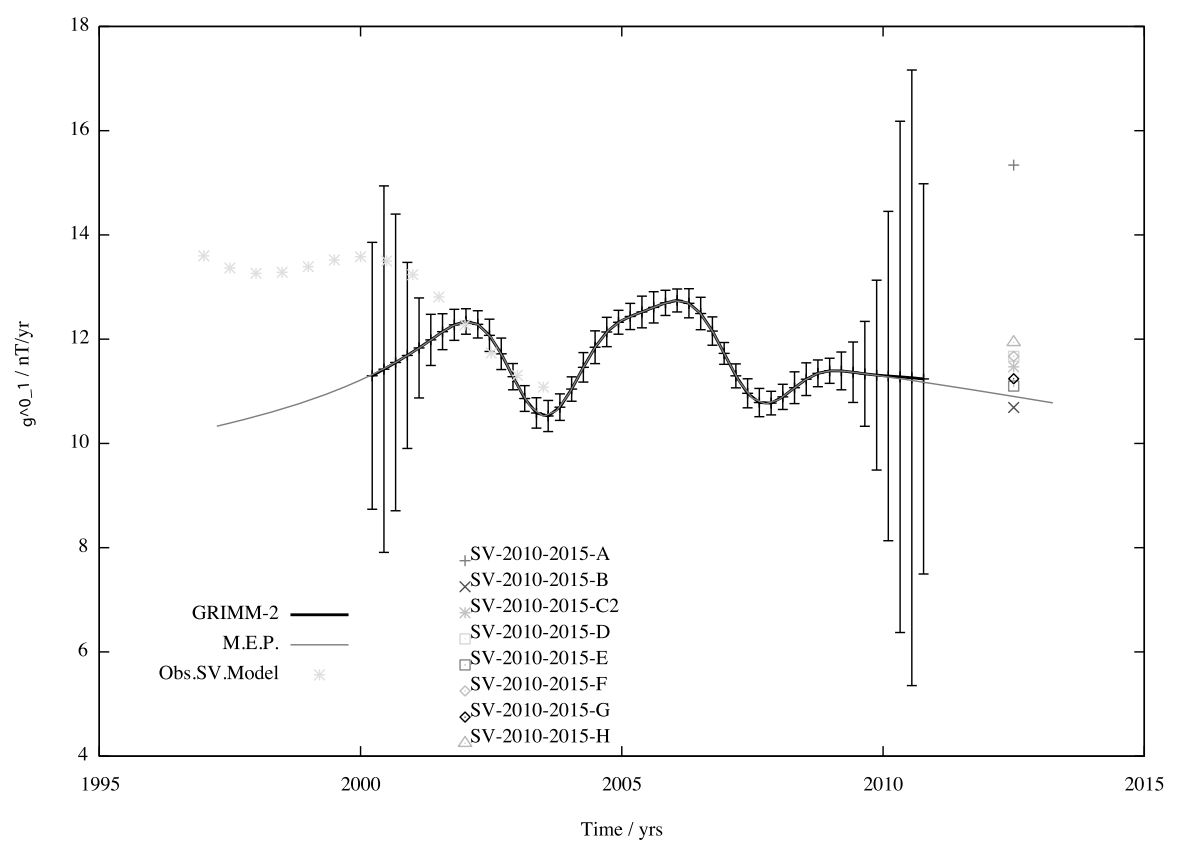

Fig. 13. Rate of change of the axial dipole (in units of nT/yr) from model GRIMM-2 (bars showing formal error estimates), along with a maximum entropy fit and extrapolation (red). A 50 year model derived from observatory monthly means (labelled Obs. SV. Model) constructed by Lesur and Wardinski (2009) is shown in stars to illustrate the rate of change prior to the validity of GRIMM-2. The remaining symbols show the predictions of the IGRF-11 SV candidates plotted for their central epoch of 2012.5.

date $\mathrm{E}$ has a number of positive and negative anomalies of amplitude $>20 \mathrm{nT} / \mathrm{yr}$ that are distributed over all latitudes. Candidates B, D, F and $\mathrm{H}$ show only minor differences from $M_{w}$. Candidate A shows global differences at Earth's surface that appear to be mainly due to a difference in its axial dipole term, but a significant difference in its equatorial dipole contribution is also evident at equatorial latitudes. Candidate $\mathrm{C} 2$ possesses differences at low latitudes and also at northern polar latitudes.

We remark that the differences between SV candidates are often most striking at low latitudes; this becomes even more obvious when the models are analyzed at the coremantle boundary. These differences amount to different predictions concerning the evolution (especially westward drift) of high amplitude flux features that are found at low latitudes at the core-mantle boundary and are responsible for a large amount of the present secular variation. Accurate determination of the evolution of these low latitude features is crucial for accurate SV predictions-it will be of great interest in the upcoming five years to see whether any of the candidates (including $\mathrm{H}$ which is based on an approximation of core physics) performs better in this regard than the weighted mean of the candidates $M_{w}$-it is unfortunately not currently possible to make a prior judgment on this matter.

4.2.4 Discussion and summary for SV-2010-2015 The decision on how best to weight the SV candidates to produce the final SV-2010-2015 model for IGRF-11 is much more challenging than in the MF scenarios because the candidates agree less well, and because there is no well established technique for accurately predicting the future evolution of the core magnetic field. The difficulties associated with field prediction are illustrated in Fig. 13. This shows in black predictions for the rate of change of the axial dipole $g_{1}^{0}$ in units of nT/yr from the GRIMM-2 timedependent field model (constrained by CHAMP satellite and observatory data between 2001.0 and 2009.5) along with formal error bars, including an extrapolation back to 2000.2 and forward to 2010.8. Also shown as a red line is a maximum entropy fit (Burg, 1967; Lacoss, 1971; Ulrych, 1972) to the GRIMM-2 model extrapolated back to 1997.25 and forward 2013.25. An assessment of the future prediction cannot yet be carried out because no observations are available. The quality of the prediction back in time can however be assessed. This is illustrated by comparison with a 50 year field model derived from observatory monthly means (Lesur and Wardinski, 2009) that is plotted here for the interval 1997.0-2003.5 and shown by the stars. The 50 year model shows the actual rate of change of $g_{1}^{0}$ in $n T / y r$ prior to 2001 lies close at the limits of the formal error bars on the extrapolation of GRIMM-2, and the maximum entropy prediction is observed to be rather poor. This is a consequence of the fundamental nonlinearity of the variations in the rate of change of the axial dipole resulting from MHD processes in the Earth's core. Looking forward to the interval 2010-2015 one can see the spread in the predictions of the SV candidate models on the right hand side of the plot at time 2012.5. The prediction of candidate A is clearly anomalous. For this reason the task force voted to disregard the prediction for $g_{1}^{0}$ (and also for $h_{1}^{1}$ ) of candidate A. Aside from this outlier the difficulty in choosing between the predictions of the candidates for the rate of change of the axial dipole is apparent.

The analyses presented earlier in this section, in both the physical and spectral domains, suggested that candidate E (which may have problems at degrees greater than 5), can- 
didate $\mathrm{G}$ (which made predictions for the sectoral harmonic different from other candidates) and candidate A (which possessed anomalous dipole terms) were consistently different from the other candidates. The task force therefore voted to allocated weights of 0.5 to $\mathrm{A}, \mathrm{E}$ and $\mathrm{G}$ with the remaining candidates $\mathrm{B}, \mathrm{C}, \mathrm{D}, \mathrm{F}, \mathrm{H}$ allocated weights of 1.0 for the construction of final IGRF-11 SV-2010-2015 model. The SV-2010-2015 model for IGRF-11 is thus identical to the model $M_{w}$ discussed previously in this section. We emphasize that in the case of SV models it is much more difficult to be certain that a particular candidate is in error simply because it differs from a mean model, because there are non-random difficulties in field forecasting, and because it is not obvious that a mean model is more likely to be correct. Further study of how best to propagate forward information from accurate MF and SV models at the current epoch is urgently needed.

\section{Conclusion}

In this article we have described some of the statistical tests carried out by the IGRF-11 task force in order to evaluate candidate models for DGRF-2005, IGRF-2010 and SV2010-2015. As a result of these tests, the task force voted in December 2009 that DGRF-2005 be composed of an unweighted combination of candidates A, B and G; that IGRF2010 be composed of candidates A, B, C2, F, G with weight 1.0 and candidates $\mathrm{D}, \mathrm{E}$ with weight 0.25 with $g_{1}^{0}$ and $h_{1}^{1}$ of candidate A discarded; and that SV-2010-2015 be composed from candidates $\mathrm{B}, \mathrm{C} 2, \mathrm{D}, \mathrm{F}, \mathrm{H}$ with weight 1.0 and candidates A, E and G with weight 0.5 , with the $g_{1}^{0}$ and $h_{1}^{1}$ $\mathrm{SV}$ predictions of candidate A discarded. The model coefficients for IGRF-11 can be found in electronic form online at http://www.ngdc.noaa.gov/IAGA/vmod/igrf.html and are also published in print in the article by Finlay et al. (2010).

The retrospective MF models submitted for DGRF-2005 were found to largely be in good agreement. Candidates A, $\mathrm{B}$ and $\mathrm{G}$, based on parent models from the established series of models CHAOS, POMME and GRIMM, were found to agree particularly well, with the formal RMS vector field error in their mean being only $1.0 \mathrm{nT}$. This close agreement is a consequence of the advances in main field modelling that have occurred in the past decade, in particular thanks to the availability of high quality satellite data from the CHAMP mission. Differences in the MF models to degree 13 are now primarily due to differences in the data selection and pre-processing strategies employed by the various teams, as well as in their choice of parameterization of external field variations. We note however that it remains possible that minor systematic errors (common to all or many candidates) could remain, for example, due to limitations in common techniques used to account for the external field variations. Improved knowledge of external fields (particularly those originating in ionospheric current systems) can be anticipated from ESA's multi-satellite constellation mission Swarm (Friis-Christensen et al., 2006) that is expected to be underway before the next IGRF revision in 2015 .

Concerning the provisional IGRF model for epoch 2010.0, differences in how teams forward propagated their estimates from mid-2009 to 2010.0, depending on the nature of the time-dependence of their parent models, was an additional source of variation between the candidates. It is clear (see Fig. 13) that accurate determination of predictive SV remains the major challenge in the IGRF process; a noticeable scatter in the submitted candidate models was again present in the IGRF-11 SV candidates and it was not possible to clearly identify one group of candidates that were demonstrably of superior quality. These difficulties were further underlined by retrospective analysis of IGRF$10 \mathrm{SV}$ candidates centered in 2007.5 which differed by 20 to $30 \mathrm{nT} / \mathrm{yr}$ from the retrospective IGRF-11 estimate for the same interval. It will be of considerable interest over the next 5 years to discover whether data assimilation methods utilizing approximations of core physics to forward propagate information (see Beggan and Whaler, 2010; Kuang et al., 2010, this issue) are yet at the stage where they can provide better forecasts than the traditional statistical extrapolation strategies.

Acknowledgments. We thank the institutes responsible for supporting the CHAMP, Ørsted and SAC-C missions for operating the satellites and making the data available. We also thank the national institutes that support ground magnetic observatories and INTERMAGNET for promoting high standards of practise. Vincent Lesur is thanked for his help in producing Fig. 13. This is IPGP contribution no. 3108 .

\section{References}

Barton, C. E., International Geomagnetic Reference Field: The Seventh Generation, J. Geomag. Geoelectr., 49, 123-148, 1997.

Beggan, C. and K. Whaler, Forecasting secular variation using core flows, Earth Planets Space, 62, this issue, 821-828, 2010.

Burg, J., Maximum entropy spectral analysis, Proc. 37th Meet. Soc. Exploration Geophysicists, 1967; reprinted in Modern Spectrum Analysis, edited by D. G. Childers, IEEE Press, New York, 1978 94, 34-39, 1967. Chambodut, A., B. Langlais, M. Menvielle, E. Thébault, A. Chulliat, and G. Hulot, Candidate models for the IGRF-11th generation making use of extrapolated observatory data, Earth Planets Space, 62, this issue, 745-751, 2010.

Chulliat, A. and E. Thébault, Testing IGRF-11 candidate models against CHAMP data and quasi-definitive observatory data, Earth Planets Space, 62, this issue, 805-814, 2010.

Constable, C. G., Parameter estimation in non-Gaussian noise, Geophys. J., 94, 131-142, 1988 .

Finlay, C. C., S. Maus, C. D. Beggan, T. N. Bondar, A. Chambodut, T. A. Chernova, A. Chulliat, V. P. Golovkov, B. Hamilton, M. Hamoudi, R. Holme, G. Hulot, W. Kuang, B. Langlais, V. Lesur, F. J. Lowes, H. Lühr, S. Macmillan, M. Mandea, S. McLean, C. Manoj, M. Menvielle, I. Michaelis, N. Olsen, J. Rauberg, M. Rother, T. J. Sabaka, A. Tangborn, L. Tøffner-Clausen, E. Thébault, A. W. P. Thomson, I. Wardinski, Z. Wei, and T. Zvereva, International Geomagnetic Reference Field: The Eleventh Generation, Geophys. J. Int., 183, 1216-1230, 2010.

Friis-Christensen, E., H. Lühr, and G. Hulot, Swarm: A constellation to study the Earth's magnetic field, Earth Planets Space, 58, 351-358, 2006.

Hamilton, B., S. Macmillan, and A. Thomson, The BGS magnetic field candidate models for the 11th generation IGRF, Earth Planets Space, 62, this issue, 737-743, 2010.

Hogg, R. V., Statistical robustness: One view of its use in applications today, Am. Stat., 33, 717-730, 1979.

Huber, P. J., Robust Statistical Procedures, SIAM, 1996.

Kuang, W., Z. Wei, R. Holme, and A. Tangborn, Prediction of geomagnetic field with data assimilation: a candidate secular variation model for IGRF-11, Earth Planets Space, 62, this issue, 775-785, 2010.

Lacoss, R. T., Data adaptive spectral analysis methods, Geophysics, 36, 661-675, 1971.

Langel, R. A. and W. J. Hinze, The Magnetic Field of the Earth's Lithosphere: The Satellite Perspective, Cambridge University Press, 1998.

Lesur, V. and I. Wardinski, A 50 years core magnetic field model under frozen-flux constraints, Geophys. Res. Abstr., 11, EGU2009-5352, 2009. 
Lesur, V., I. Wardinski, M. Hamoudi, and M. Rother, The second generation of the GFZ Reference Internal Magnetic Model: GRIMM-2, Earth Planets Space, 62, this issue, 765-773, 2010.

Lowes, F. J., Mean-square values on the sphere of spherical harmonic vector fields, J. Geophys. Res., 71, 2179, 1966.

Lowes, F. J., Spatial power spectrum of the main geomagnetic field, Geophys. J. R. Astron. Soc., 36, 717-730, 1974.

Lowes, F. J., An estimate of the errors of the IGRF/DGRF field 1945-2000, Earth Planets Space, 52, 1207-1211, 2000.

Macmillan, S. and C. C. Finlay, The International Geomagnetic Reference Field, IAGA Sopron Book Series, 2010 (in press).

Maus, S., S. Macmillan, F. J. Lowes, and T. Bondar, Evaluation of candidate geomagnetic field models for the 10th generation of IGRF, Earth Planets Space, 57, 1173-1181, 2005.

Maus, S., C. Manoj, J. Rauberg, I. Michaelis, and H. Lühr, NOAA/NGDC candidate models for the 11th generation International Geomagnetic Reference Field and the concurrent release of the 6th generation Pomme magnetic model, Earth Planets Space, 62, this issue, 729-735, 2010.

Olsen, N., A model of the geomagnetic field and its secular variation for epoch 2000 estimated from Ørsted data, Geophys. J. Int., 149, 454-462,
2002

Olsen, N., M. Mandea, T. J. Sabaka, and L. Tøffner-Clausen, The CHAOS3 geomagnetic field model and candidates for the 11th generation IGRF, Earth Planets Space, 62, this issue, 719-727, 2010.

Silva, L., S. Maus, G. Hulot, and E. Thébault, On the possibility of extending the IGRF predictive secular variation model to a higher SH degree, Earth Planets Space, 62, this issue, 815-820, 2010.

Thébault, E., A. Chulliat, S. Maus, G. Hulot, B. Langlais, A. Chambodut, and M. Menvielle, IGRF candidate models at times of rapid changes in core field acceleration, Earth Planets Space, 62, this issue, 753-763, 2010.

Ulrych, T., Maximum entropy power spectrum of long period geomagnetic reversals, Nature, 235, 218-219, 1972.

Winch, D. E., D. J. Ivers, J. P. R. Turner, and R. J. Stening, Geomagnetism and Schmidt quasi-normalization, Geophys. J. Int., 149, 487-504, 2004.

C. C. Finlay (e-mail: cfinlay@erdw.ethz.ch), S. Maus, C. D. Beggan, M. Hamoudi, F. J. Lowes, N. Olsen, and E. Thébault 